\title{
Energy and Intensity Calibration Standards
}

by

R. B. Firestone 


\section{DISCLAIMER}

This report was prepared as an account of work sponsored by an agency of the United States Government. Neither the United States Government nor any agency Thereof, nor any of their employees, makes any warranty, express or implied, or assumes any legal liability or responsibility for the accuracy, completeness, or usefulness of any information, apparatus, product, or process disclosed, or represents that its use would not infringe privately owned rights. Reference herein to any specific commercial product, process, or service by trade name, trademark, manufacturer, or otherwise does not necessarily constitute or imply its endorsement, recommendation, or favoring by the United States Government or any agency thereof. The views and opinions of authors expressed herein do not necessarily state or reflect those of the United States Government or any agency thereof. 


\section{DISCLAIMER}

Portions of this document may be illegible in electronic image products. Images are produced from the best available original document. 
The following table of energy and intensity standards includes isotopes with a proven track record at this laboratory and elsewhere, as well as isotopes which offer the possibility of future application. Data is also included for some of those trespassing isotopes which appear in the background or as common impurities in experiments, and which might be useful for calibration. It is likely that other isotopes not reported here can be useful, and some of those mentioned may have only special application due to half-life or other considerations.

Energy and intensity values presented in this table are chosen by various critereon. Energles measured by bent crystal spectrometer were 1 usually given stronger consideration, although certain $\mathrm{Ge}(\mathrm{L} 1)$ work (e.g. Idaho Falls) was given great weight. No effort is made to average different values, and given error estimates were weighted strongly in choosing energies. Efficiency. data is much more difficult to judge, and it was usually considered most feasible to choose all values from one author. Given errors were not used as a strong critereon, in general, because it is questionable whether these errors contain more than statistical fluctuations. In addition many authors clearly miscalculated their errors. The track record of certain groups was given added weight (e.g. Livermore) as was the date the work was completed (more recent work is favored). Additional references are provided for those who wish to follow up the available measurements on a given isotope, but no attempt at completeness is made, and older work is 
generally referenced in Lederer's Table of the Isotopes, 6th Ed., (Led68).

An index of $\gamma$-ray standards. is supplied to provide quick reference to the isotopes in this table. Half-lives and suggested means of production are presented, as well as the energy range covered:by the isotope. The standard is delineated into general categories as to whether it contains primary or secondary quality measurements, and the numbers of each. Primary energy. standards have high accuracy and are usually better than $0.01 \mathrm{keV}$ at low energies and $0.05 \mathrm{keV}$ at higher energies. Secondary energy standards are slight1y less accurate, but are usually better than $0.10 \mathrm{keV}$. Primary intensity standards were measured by painstaking methods or involved simple decay systematics which lead to very accurate values. Secondary intensity standards were usually measured with the primary standards. The primary and secondary classifications are a bit arbitrary and are used only as a general guideline.

Some of the values in this table will be subject to change as new, better techniques are applied to the measurements. No warrantee is implied to any of the numbers in this table, and the user is advised to be careful in accepting any numbers or errors with less than a jaundiced eye. Standard calibration is a difficult game, and even veteran experimenters may. miss a measurement. The author w111 appreciate updated information for inclusion in this table as well as any corrections or suggestions. 
Index of $\gamma$-ray Standards Compiled

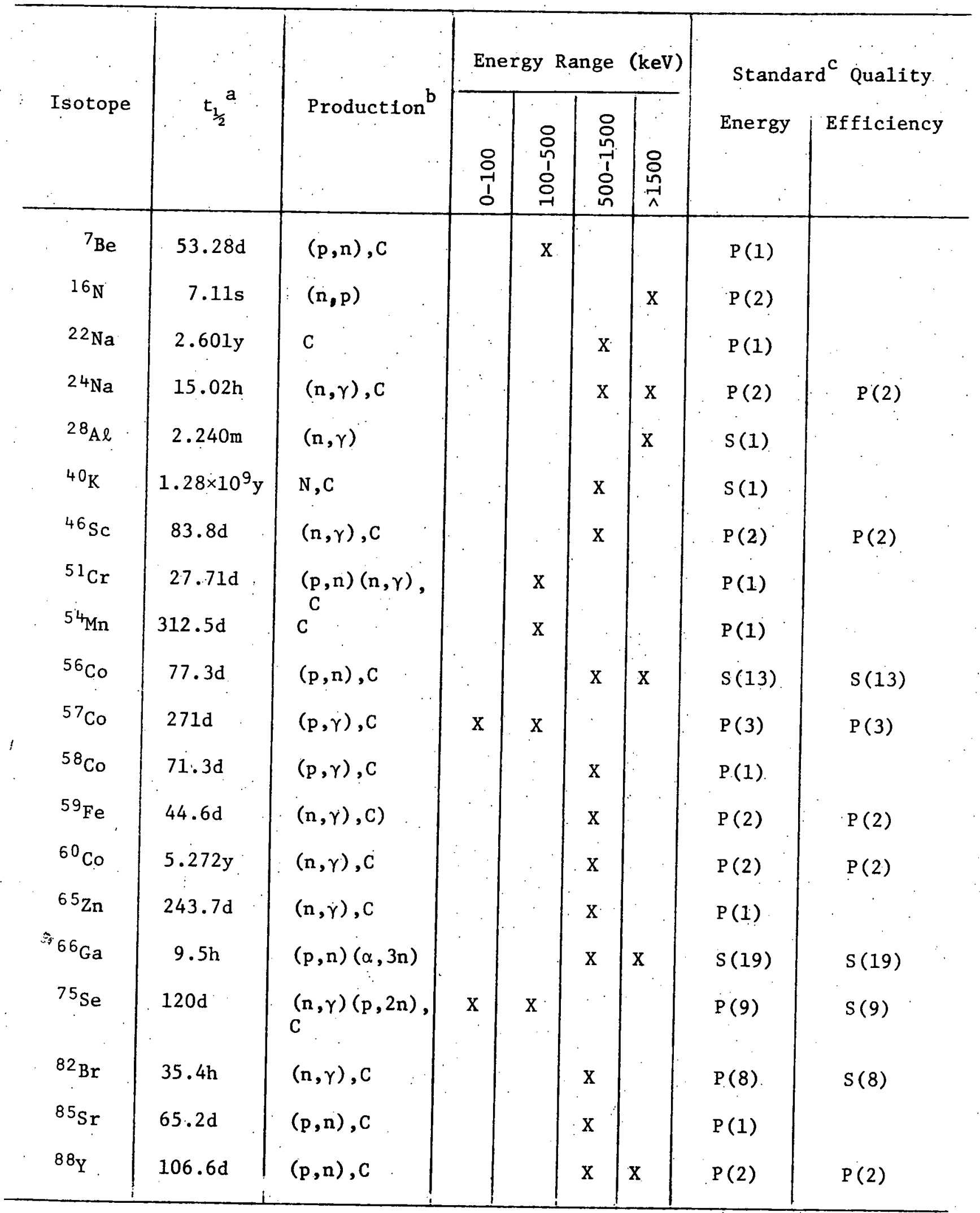

(Continued)... 


\begin{tabular}{|c|c|c|c|c|c|c|c|c|}
\hline $90 m_{Y}$ & $64.0 \mathrm{~h}$ & $(n, \gamma), C$ & & $\mathrm{X}$ & & & $P(2)$ & P.(2) \\
\hline${ }^{94} \mathrm{Nb}$ & $2.0 \times 10^{4} y$ & $(n, \gamma), c$ & & & $\mathrm{X}$ & & $P(2)$ & $P(2)$ \\
\hline${ }^{95} \mathrm{Zr}$ & $65.5 \mathrm{~d}$ & $(n, \gamma), C$ & & & $\dot{x}$ & & $P(2)$ & \\
\hline${ }^{95} \mathrm{Nb}$ & $35.1 d$ & $\left(B^{-}\right), C$ & & & $\mathrm{x}$ & & $P(1)$ & \\
\hline $95 m_{\mathrm{Tc}}$ & $61 d$ & $(p, n)$ & & $\mathrm{X}$ & $\mathrm{x}$ & & $\dot{P}(5)$ & $s(7)$ \\
\hline${ }^{99} \mathrm{Mo}$ & $66.02 \mathrm{~h}$ & $(n, \gamma), c$ & $\mathrm{x}$ & $\mathrm{x}$ & $\mathrm{x}$ & & $P(5)$ & $s(6)$ \\
\hline $108 m_{\mathrm{Ag}}$ & $1.3 \times 10^{2} y$ & $(n, \gamma)$ & & $\mathrm{X}$ & $\mathrm{x}$ & & $s(3)$ & $P(3)$ \\
\hline${ }^{109} \mathrm{Cd}$ & $453 d$ & $(p, n), C$ & $x$ & & & & $P(1)$ & \\
\hline $110 m_{\mathrm{Ag}}$ & $252 d$ & $(n, \gamma), C$ & & $\mathrm{X}$ & $\mathrm{x}$ & $\mathrm{x}$ & $P(9)$ & $S(15)$ \\
\hline $113 \mathrm{Sn}$ & $115 \mathrm{~d}$ & $(\underset{c}{p}, 3 n)(n, \gamma)$, & & $\mathrm{x}$ & . & & $P(1)$ & \\
\hline $124 \mathrm{Sb}$ & $60.20 d$ & $(n, \gamma), c$ & & & $\mathrm{X}$ & $\mathrm{x}$ & $P(9)$ & $S(11)$ \\
\hline $125_{\mathrm{Sn}}$ & $9.65 \mathrm{~d}$ & $(n, \gamma)$ & & $\mathrm{x}$ & $\mathrm{x}$ & $x$ & $P(3)$ & \\
\hline${ }^{132} \mathrm{Cs}$ & $6.47 \mathrm{~d}$ & $(p, n)$ & & $\mathrm{X}$ & $\mathrm{X}$ & & $P(1)$ & $\dot{S}(8)$. \\
\hline $133_{\mathrm{Ba}}$ & $10.4 \mathrm{y}$ & c & $\mathrm{x}$ & $\mathrm{x}$ & & & & $S(5)$ \\
\hline $13{ }^{4} \mathrm{Cs}$ & $2.06 \mathrm{y}$ & $(n, \gamma), c$ & & $\mathrm{X}$ & $\dot{x}$ & & & \\
\hline $137 \mathrm{Cs}$ & $30: 1$ & C & . & & $\mathrm{X}$ & & $P(1)$ & \\
\hline${ }^{139} \mathrm{Ce}$ & $137.5 \mathrm{~d}$ & $(p, n), C$ & & $\mathrm{x}$ & & & $P(1)$ & \\
\hline${ }^{141} \mathrm{Ce}$ & $32.53 \mathrm{~d}$ & $(n, \gamma), c$ & & $\mathrm{x}$ & & & $P(1)$ & \\
\hline${ }^{152} \mathrm{Eu}$ & $12 \mathrm{y}$ & $(n, \gamma), c$ & & $x$ & $\mathrm{X}$ & & & $S(12)$ \\
\hline $153_{G d}$ & $241.5 d$ & c & $\mathrm{x}$ & $\mathrm{X}$ & & & $P(6)$ & $S(9)$ \\
\hline $154 \mathrm{Eu}$ & $8.6 y$ & $(n, \gamma), C$ & & $\mathrm{x}$ & $\mathrm{x}$ & & $P(11)$ & \\
\hline $155_{\mathrm{Eu}}$ & $4.8 y$ & $\mathrm{C}$ & $\mathrm{x}$ & $\mathrm{x}$ & & & $P(5)$ & $S(4)$ \\
\hline${ }^{160} \mathrm{~Tb}$ & $72.3 d$ & $(n, \gamma), c$ & $\mathrm{x}$ & $\mathrm{x}$ & $\mathrm{x}$ & & $P(12)$ & $S(14)$ \\
\hline $.166 m_{\mathrm{Ho}}$ & $1.2 \times 10^{3} \mathrm{y}$ & $(n, \gamma), c$ & $\mathrm{X}$ & $\mathrm{x}$ & $x$ & & & $S(24)$ \\
\hline${ }^{169} \mathrm{Yb}$ & $31 d$ & $(p, n), c$ & $\mathrm{x}$ & $\mathrm{x}$ & & & $P(2)$ & $S(11)$ \\
\hline $170^{\mathrm{Tm}}$ & $129 d$ & $(n, \gamma), c$ & $x$ & & & & $P(1)$ & \\
\hline $177 m_{\mathrm{Lu}}$ & $161.0 \mathrm{~d}$ & $(n, \gamma)$ & $\mathrm{X}$ & $\mathrm{x}$ & & & $S(30)$ & $S(32)$ \\
\hline
\end{tabular}




\begin{tabular}{|c|c|c|c|c|c|c|c|c|}
\hline${ }^{182} \mathrm{Ta}$ & $115 \mathrm{~d}$ & $(n, \gamma), c$ & $\mathrm{x}$ & $\mathrm{x}$ & $\mathrm{X}$ & & $P(30)$ & $S(24)$ \\
\hline $183^{3} \mathrm{Ta}$ & $5.0 \mathrm{~d}$ & $\mathrm{C}$ & $\mathrm{x}$ & $\mathrm{x}$ & & & $P(12)$ & S.(12) \\
\hline $1850 \mathrm{~s}$ & $84 d$ & $(p, n)$ & & & $\mathrm{X}$ & & $P(5)$ & $S(5)$ \\
\hline${ }^{1910 s}$ & $.15 .3 d$ & $(n, \gamma), c$ & $\mathrm{X}$ & $\mathrm{X}$ & & & $P(2)$ & . \\
\hline${ }^{192} \mathrm{Ir}$ & $74.3 d$ & $(n, \gamma), C$ & & $\mathrm{X}$ & $\mathrm{x}$. & & $P(9)$ & $S(11)$ \\
\hline - $198 \mathrm{Au}$ & $2.695 d$ & $(n, \gamma), c$ & & $\mathrm{x}$ & $\mathrm{X}$ & . & $P(3)$ & $S(3)$ \\
\hline $20{ }^{3} \mathrm{Hg}$ & $46.60 \mathrm{~g}$ & $(n, \gamma), c$ & . & $\mathrm{x}$ & & & $P(1)$ & \\
\hline $207_{\mathrm{Bi}}$ & $38 y$ & C & & $\mathrm{x}$ & $\mathrm{X}$ & $\mathrm{X}$ & $P(3)$ & $S(3)$ \\
\hline${ }^{226} \mathrm{Ra}$ & $1.6 \times 10^{2} y$ & C & $\mathrm{X}$ & $\mathrm{X}$ & $\mathrm{x}$ & $\mathrm{X}$ & $S(23)$ & S (23) \\
\hline${ }^{228} \mathrm{Th}$ & $1.913 y$ & $\mathrm{C}$ & & $\mathrm{x}$ & $\mathrm{x}$ & $\mathrm{X}$ & $P(3)$ & $S(3)$ \\
\hline${ }^{241} \mathrm{Am}$ & $435 y$ & C & $\mathrm{X}$ & & & & $P(2)$ & $S(2)$ \\
\hline $24^{3} \mathrm{Cm}$ & $28 y$ & $\mathrm{C}$ & $\mathrm{x}$ & $\mathrm{X}$ & & & & $S(4)$ \\
\hline
\end{tabular}
a) Chart of Nuclides, Knolls Atomic Power Laboratory, 11th Ed., 1972.
b) Mode(s) of production, $\mathrm{C}=$ commercially avallable, $\mathrm{N}$ = naturally abundant.
c) $\mathrm{P}=$ primary standard (extreme accuracy); $\mathrm{S}=$ secondary standard. Number of useful transitions are given in parenthesis. 
$\gamma$-Ray Energy and Intensity Standards

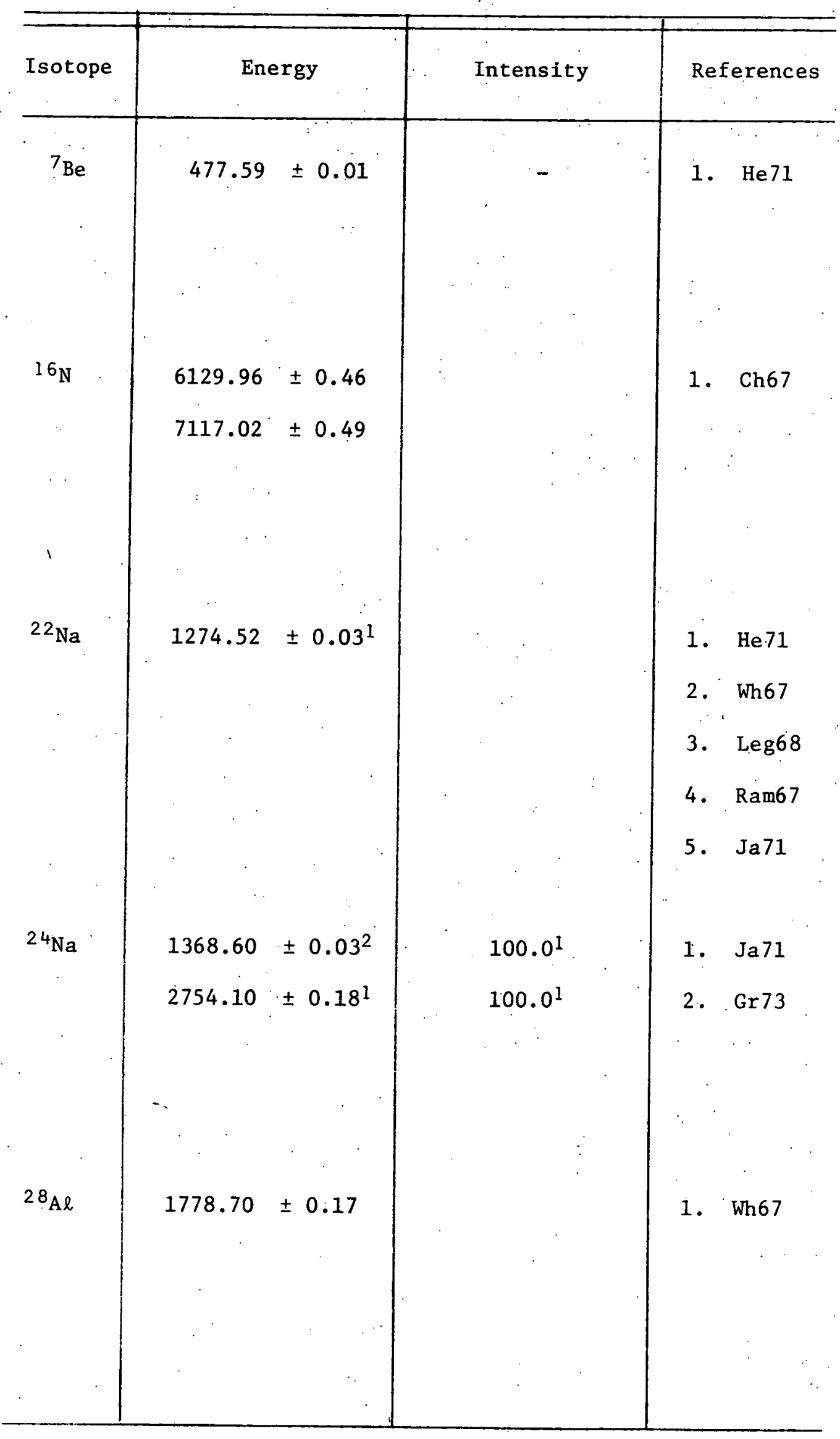




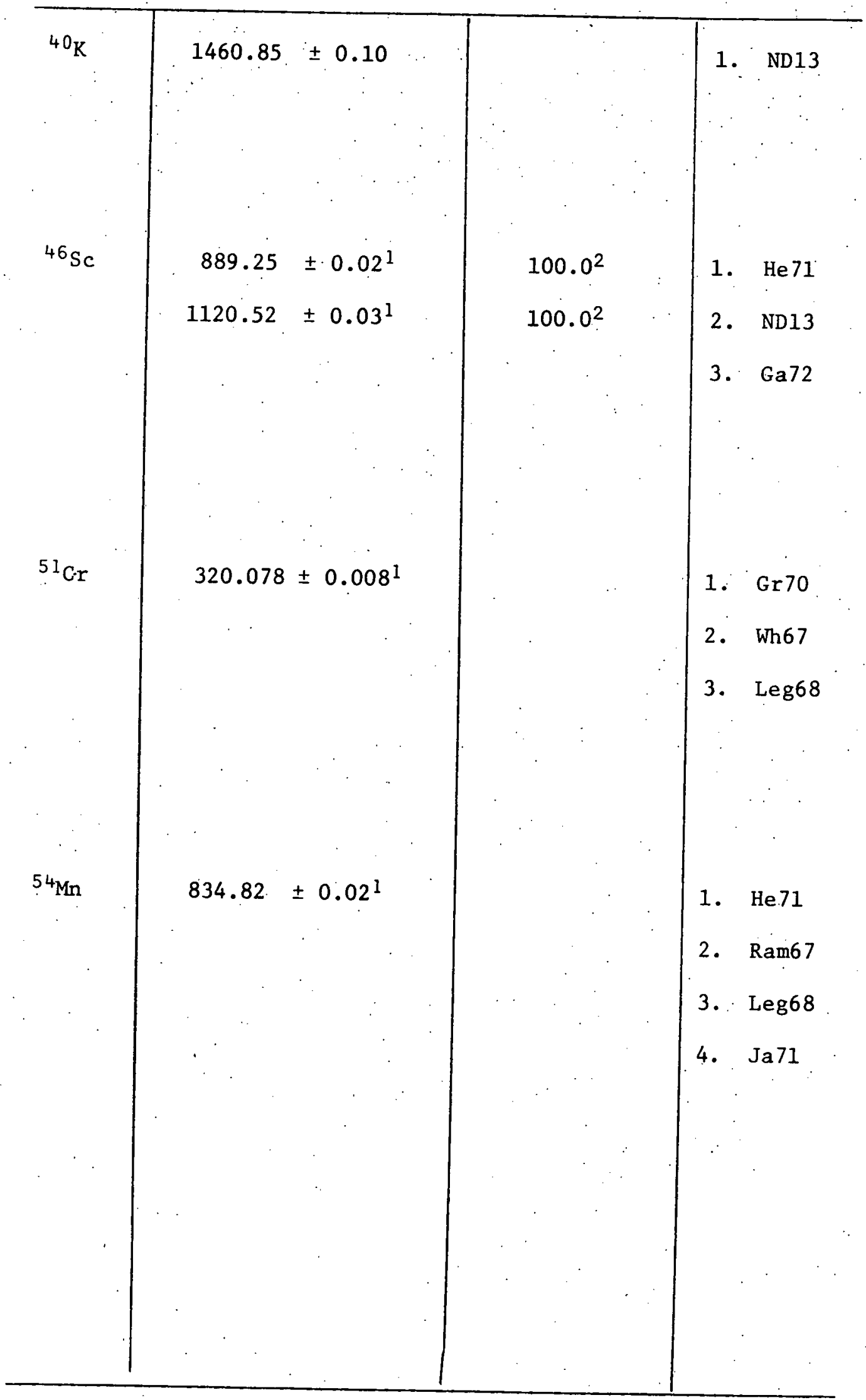




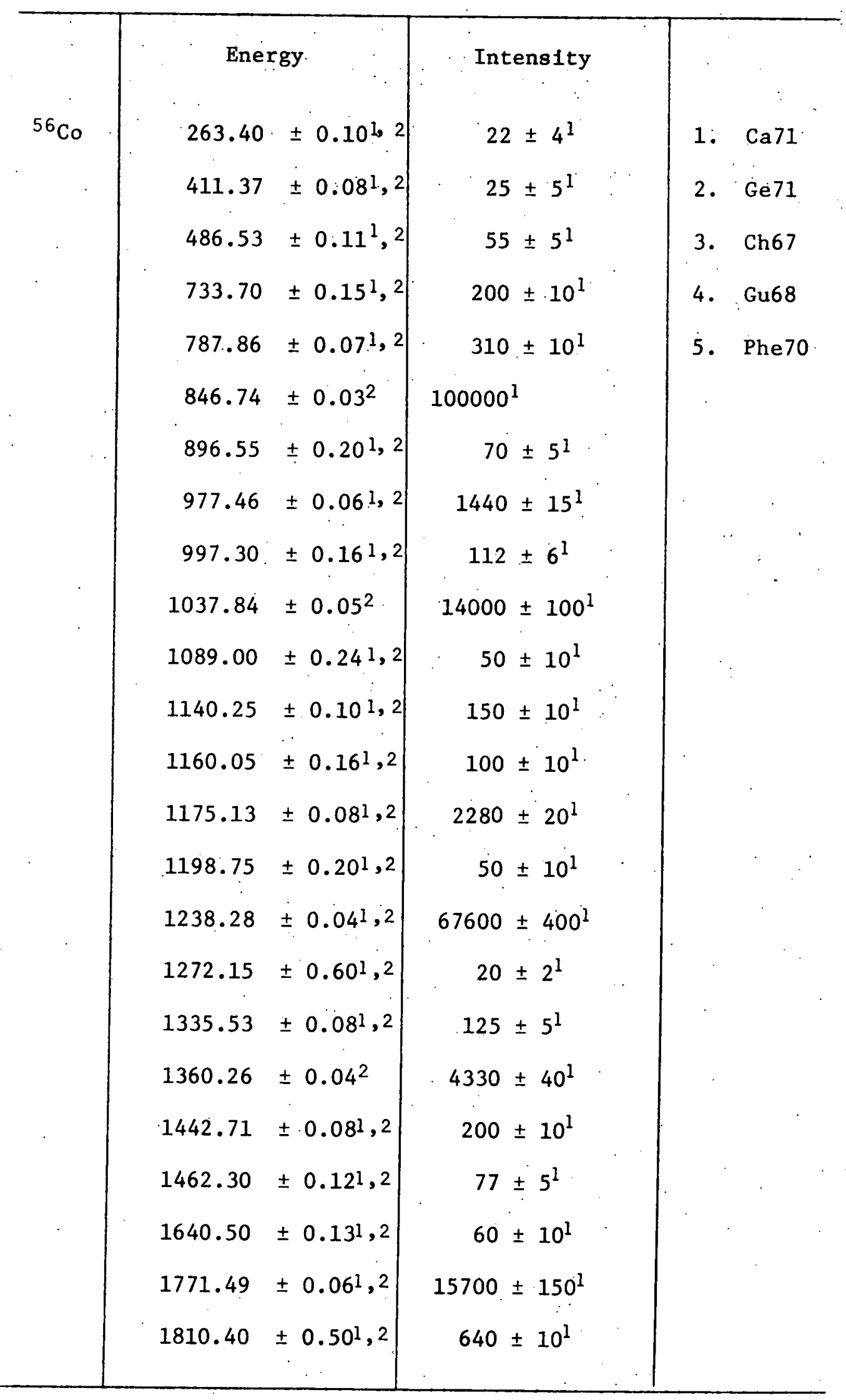




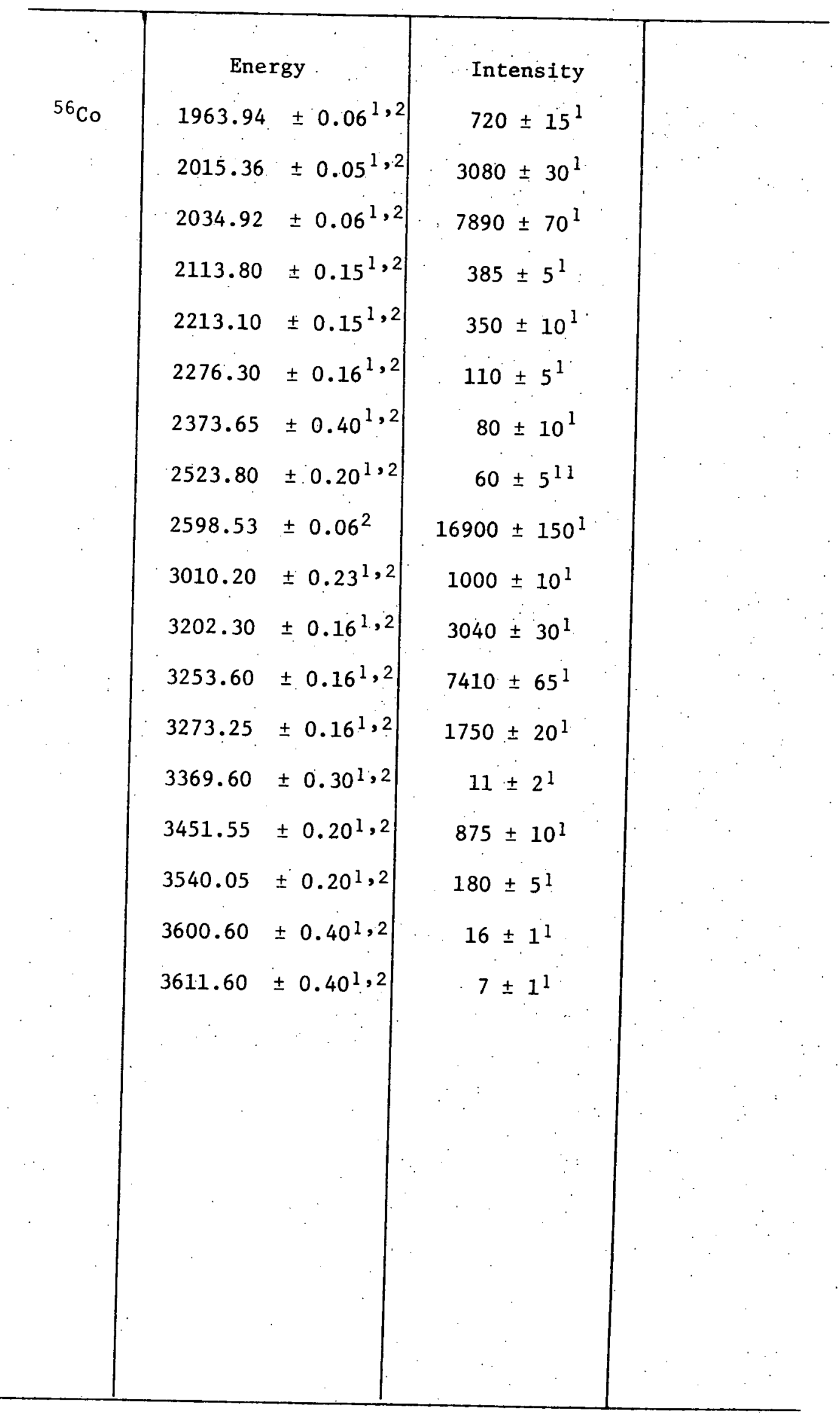




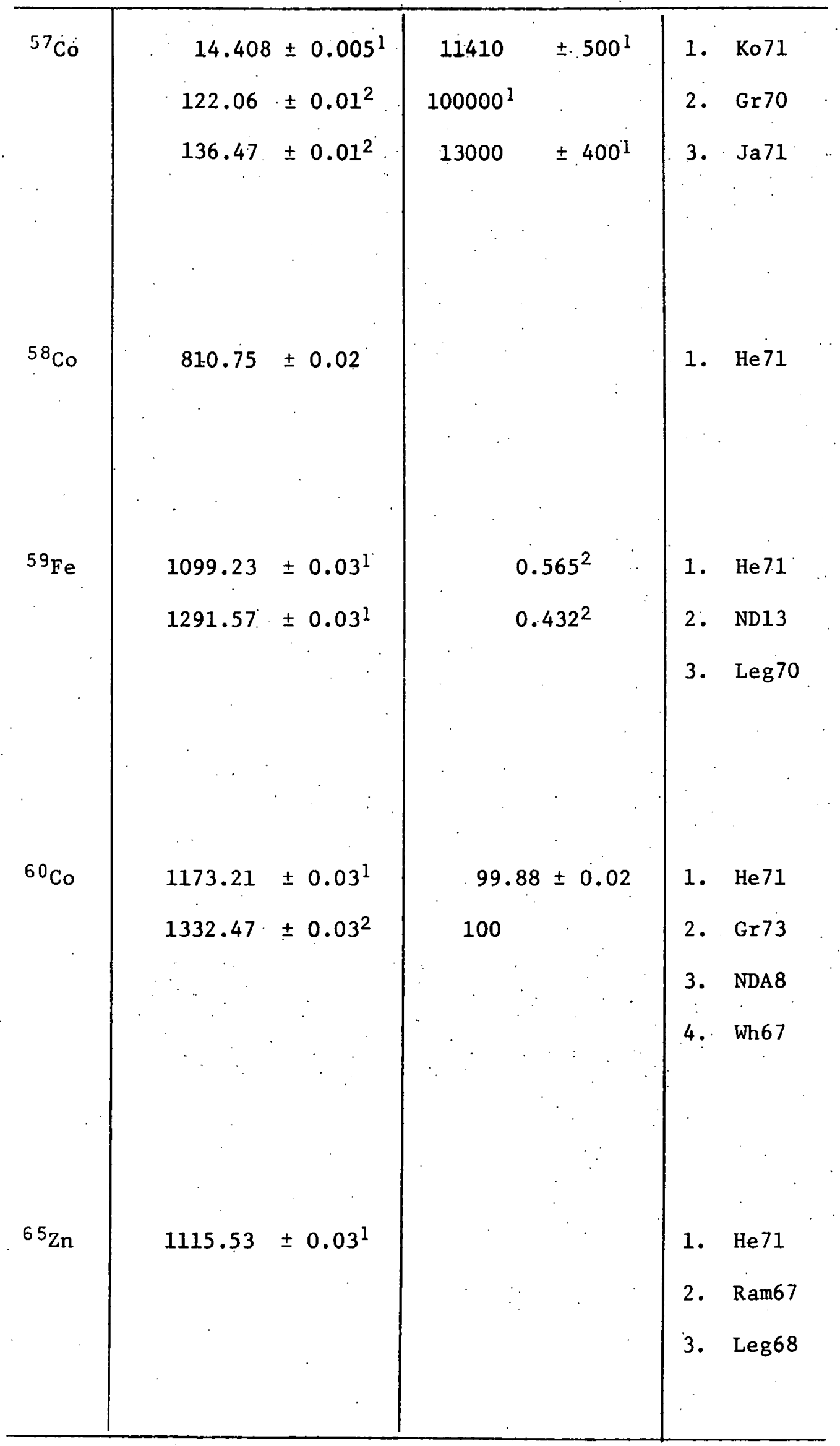




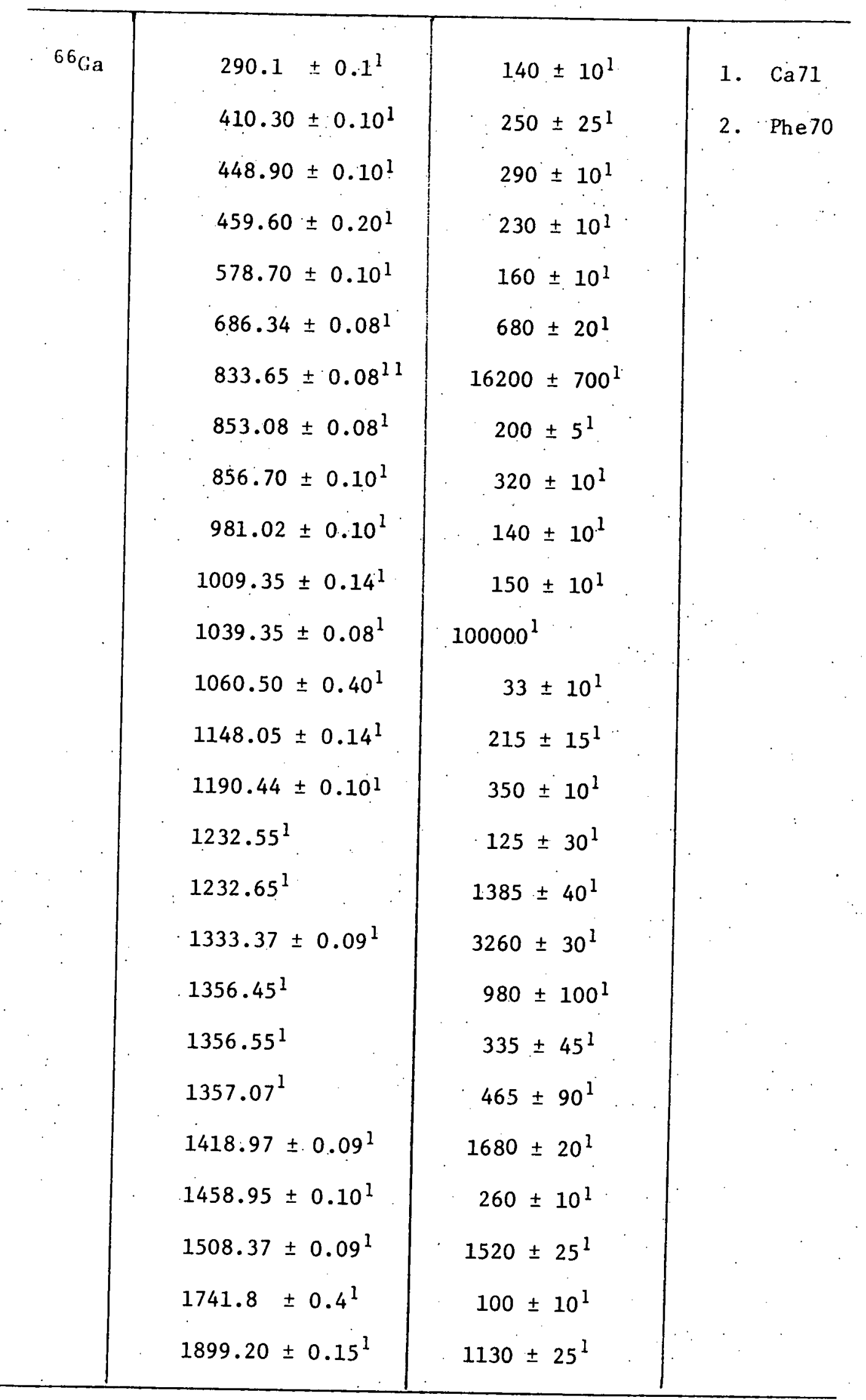




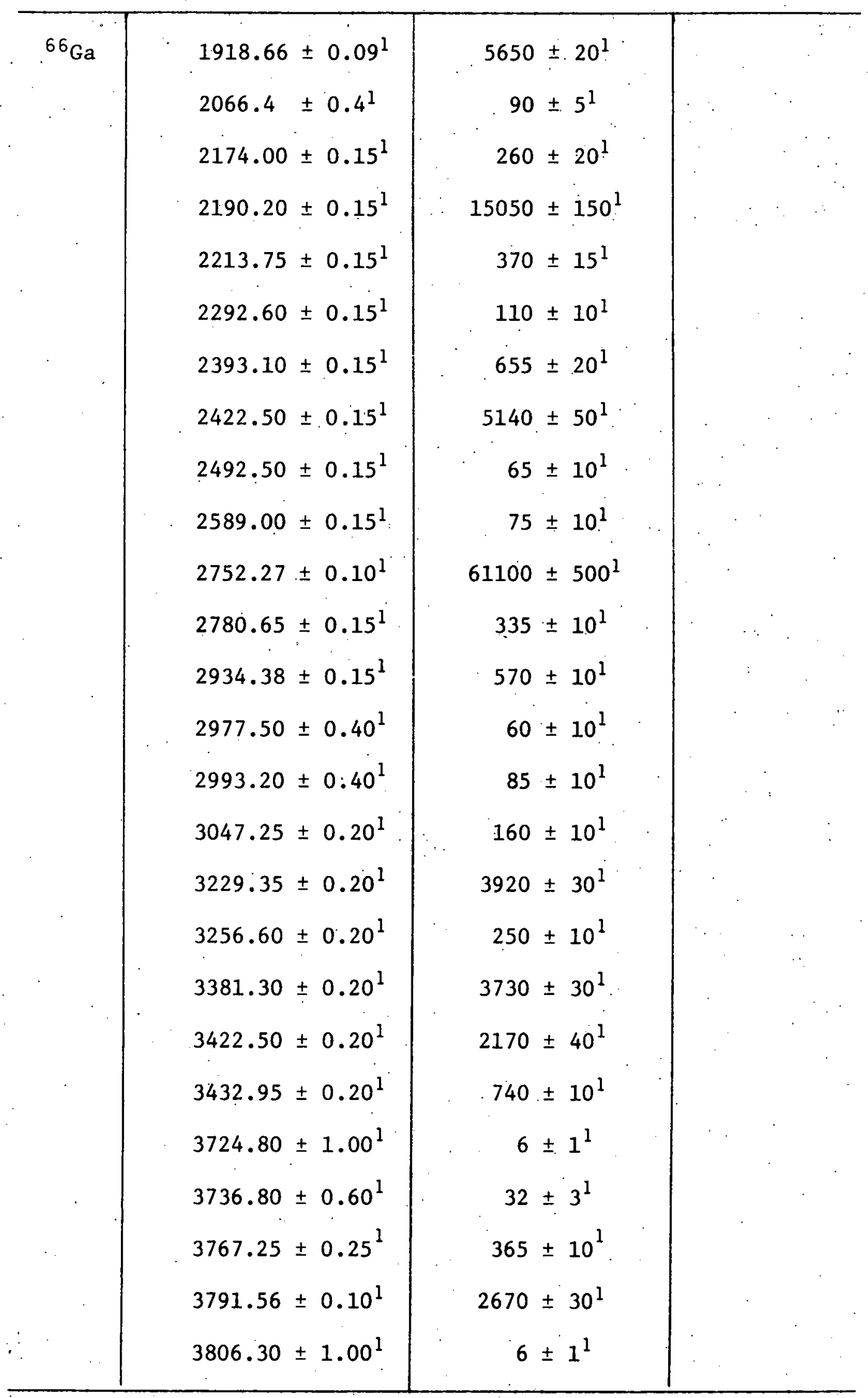




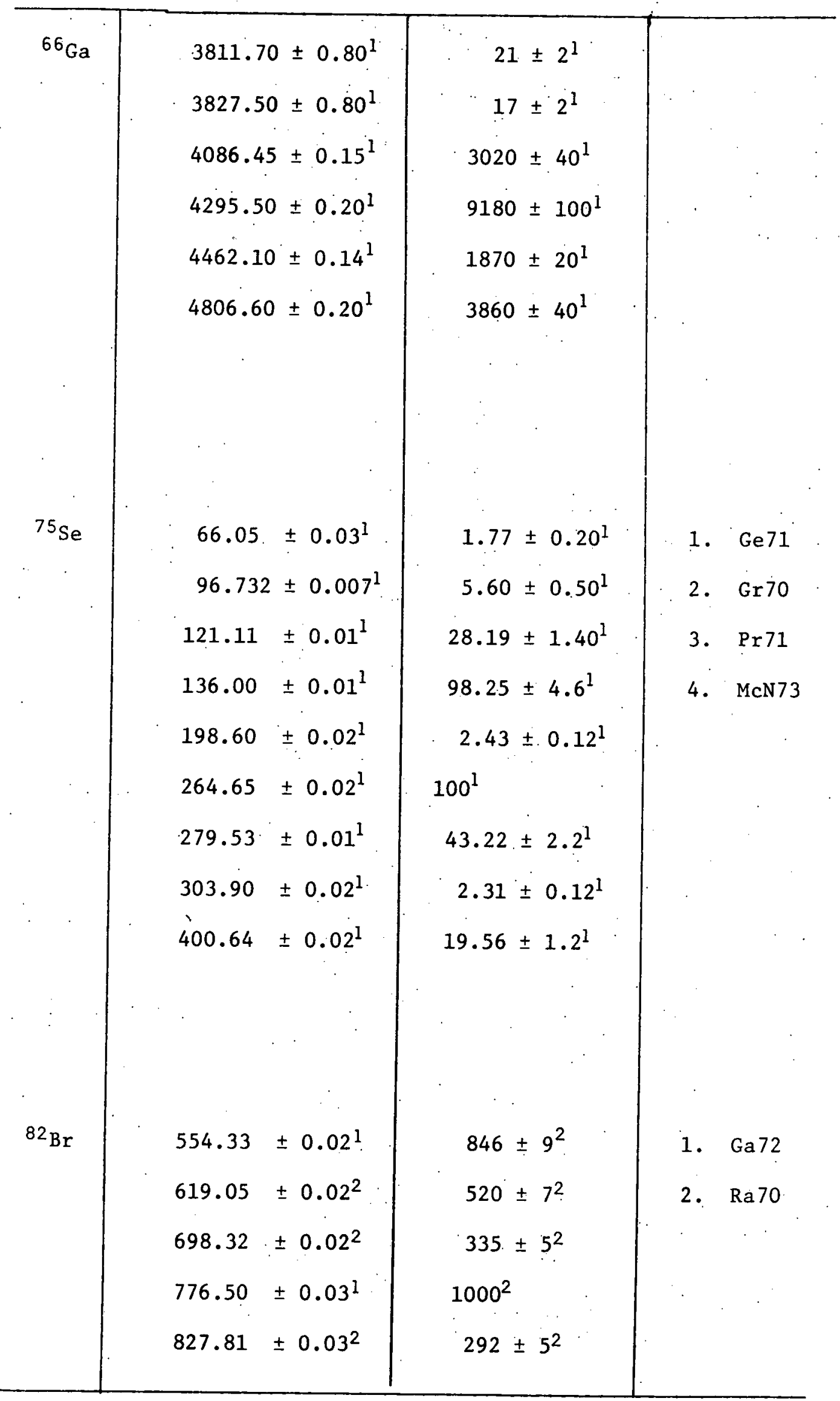




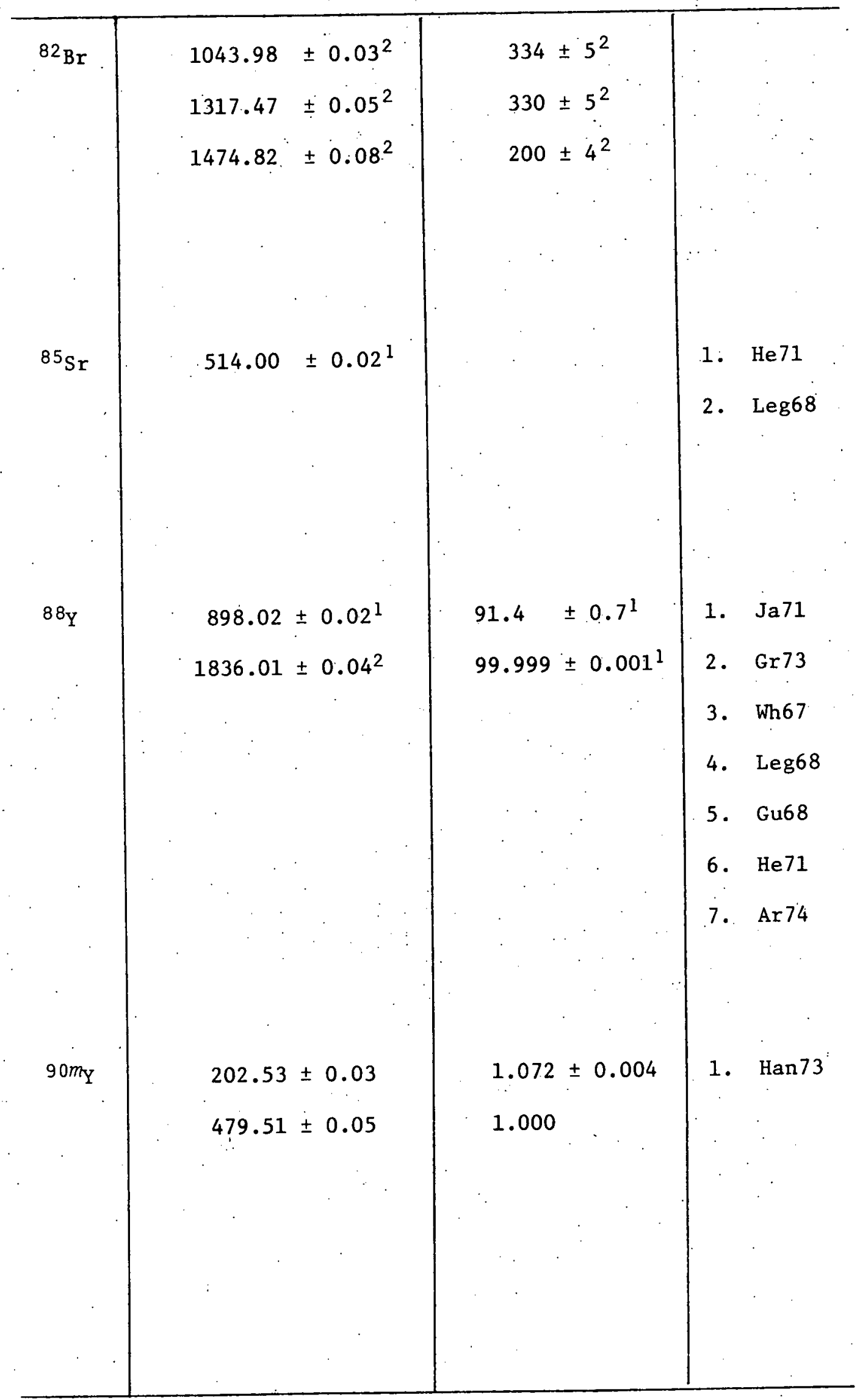




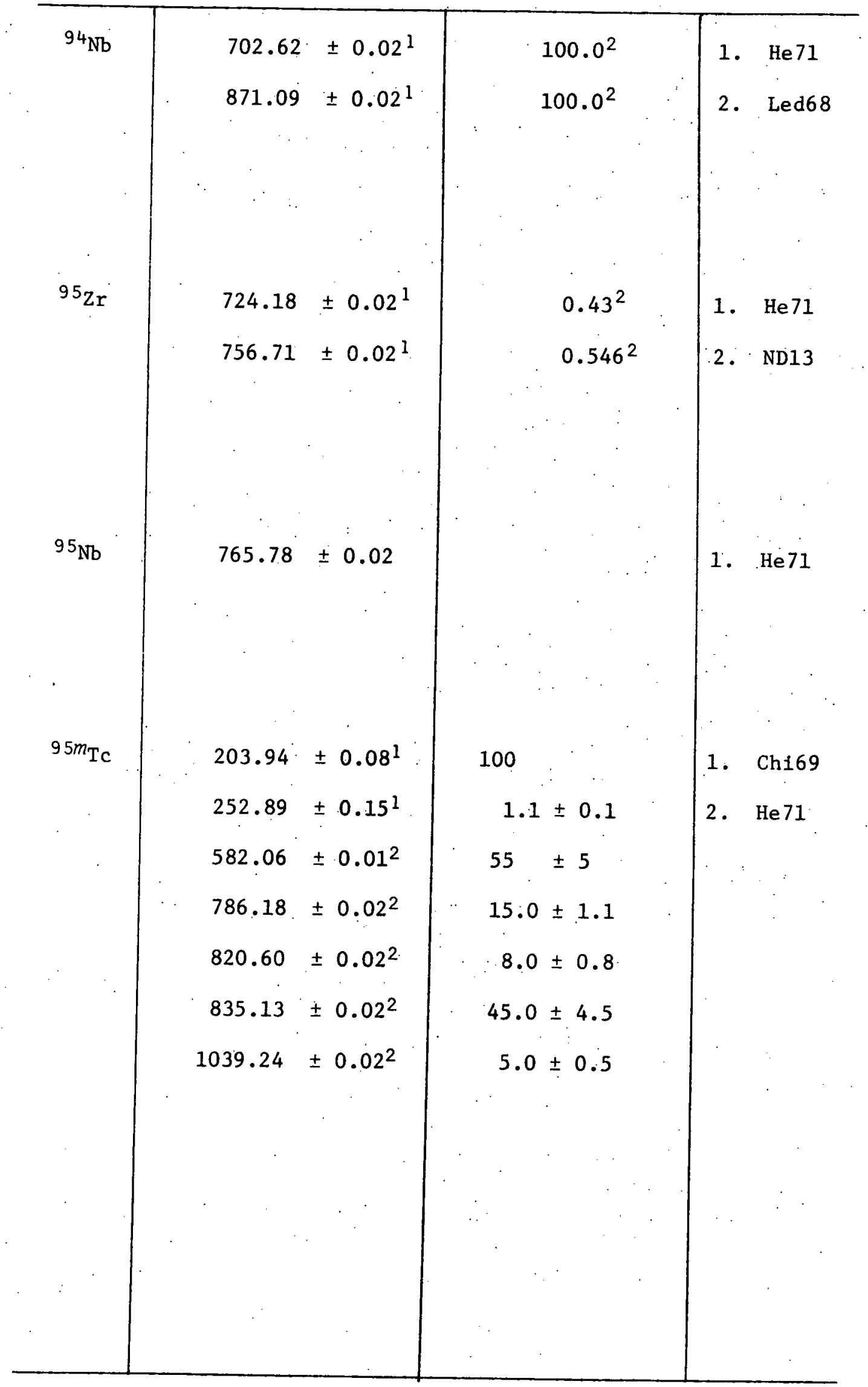




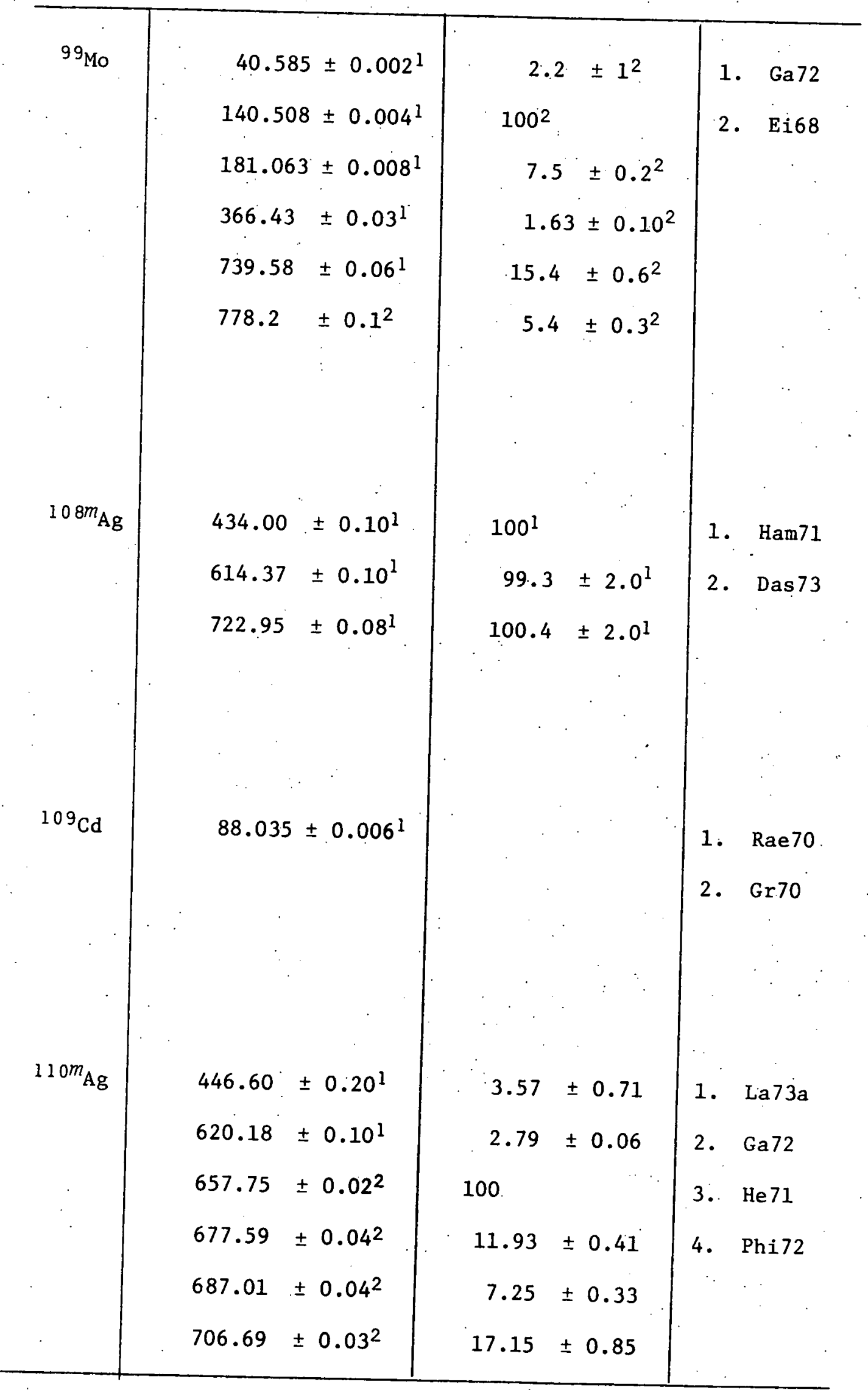




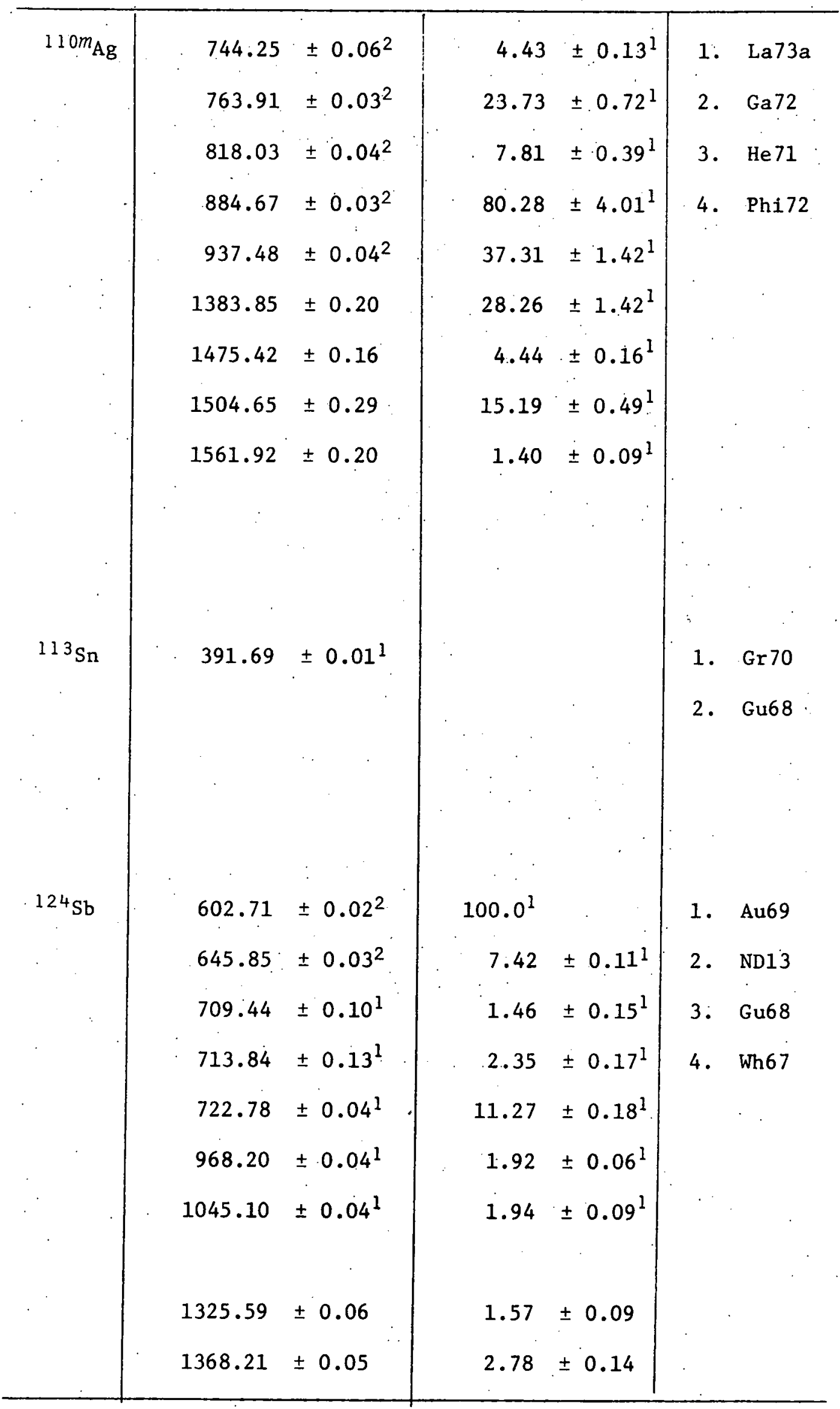




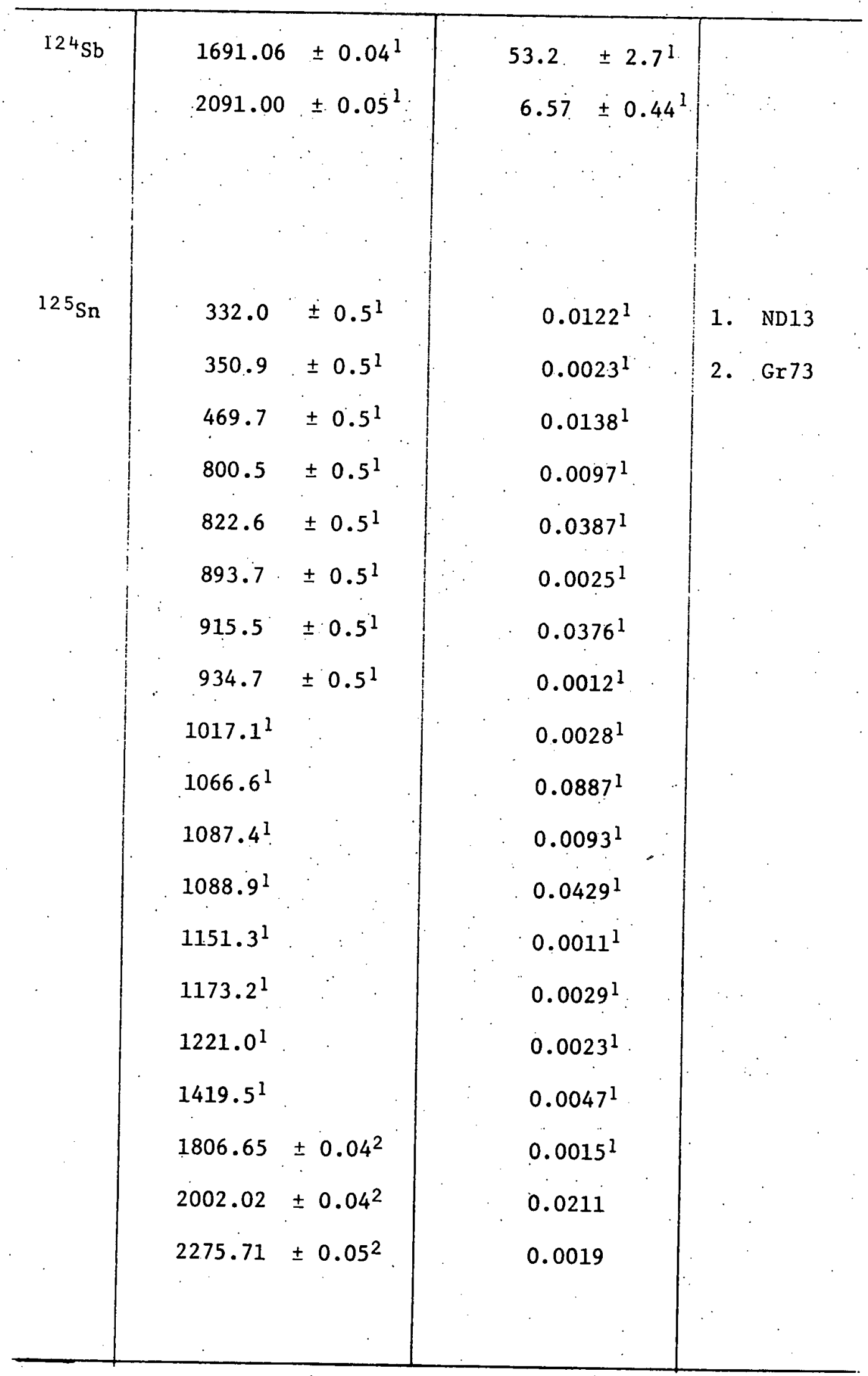




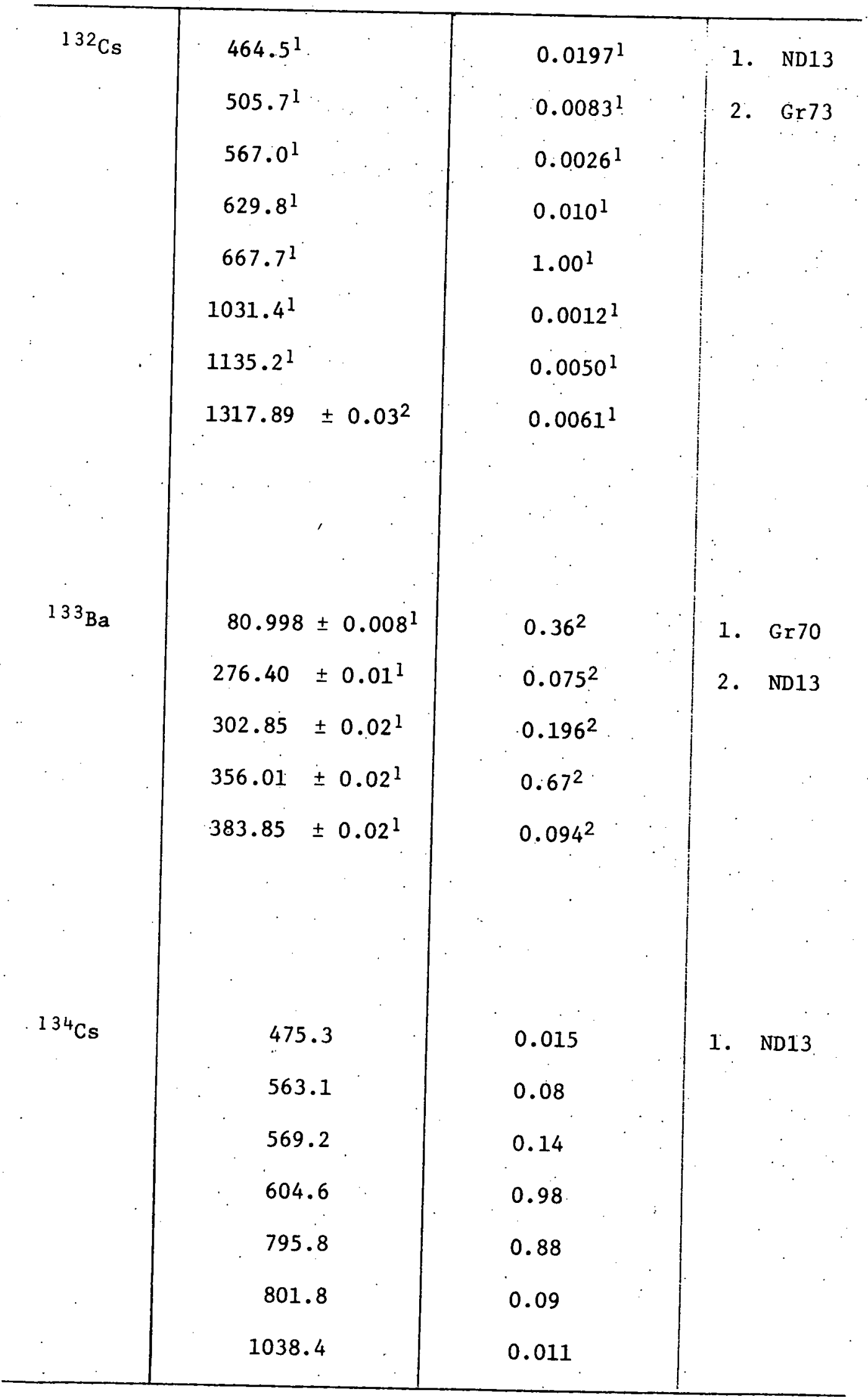




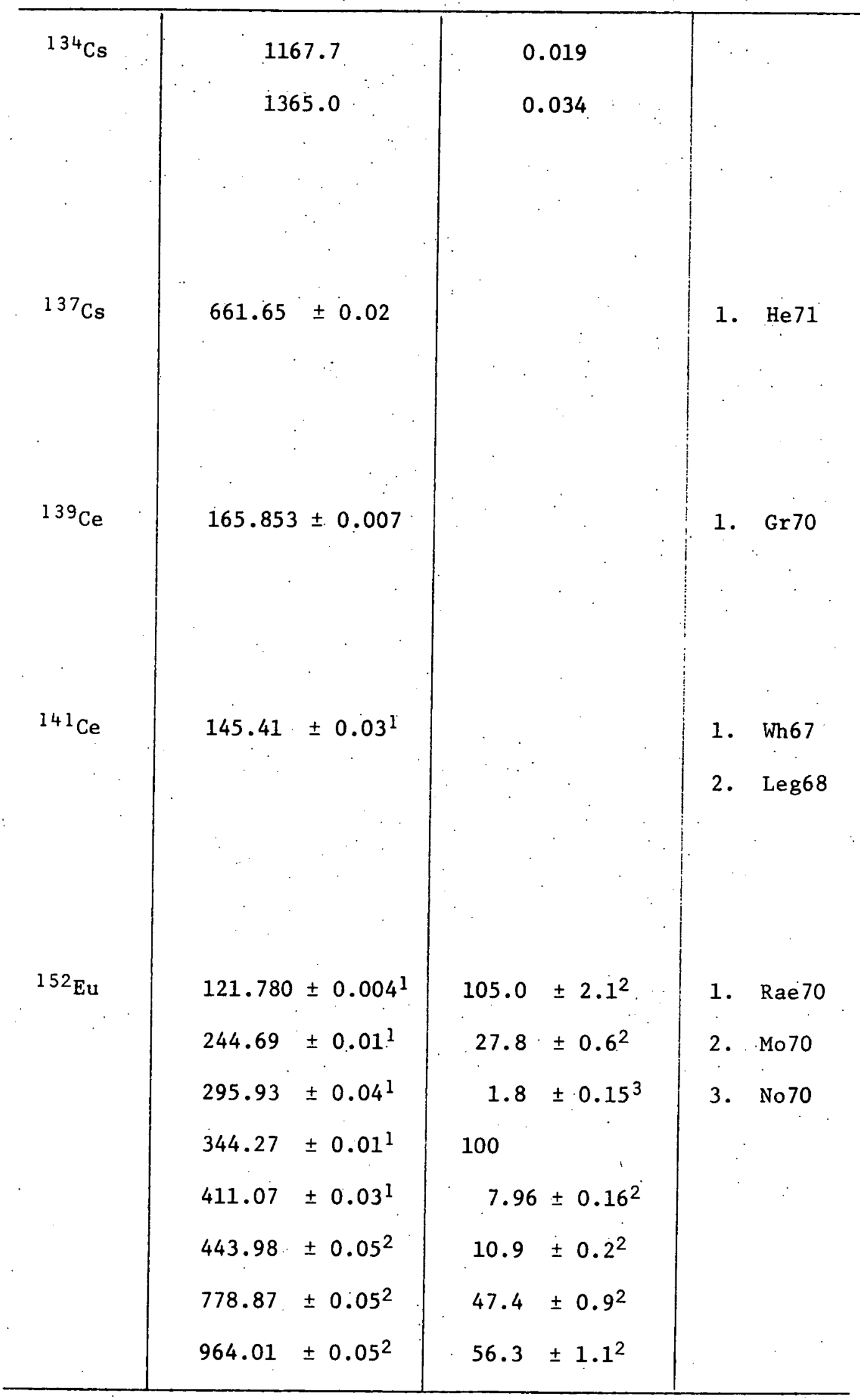




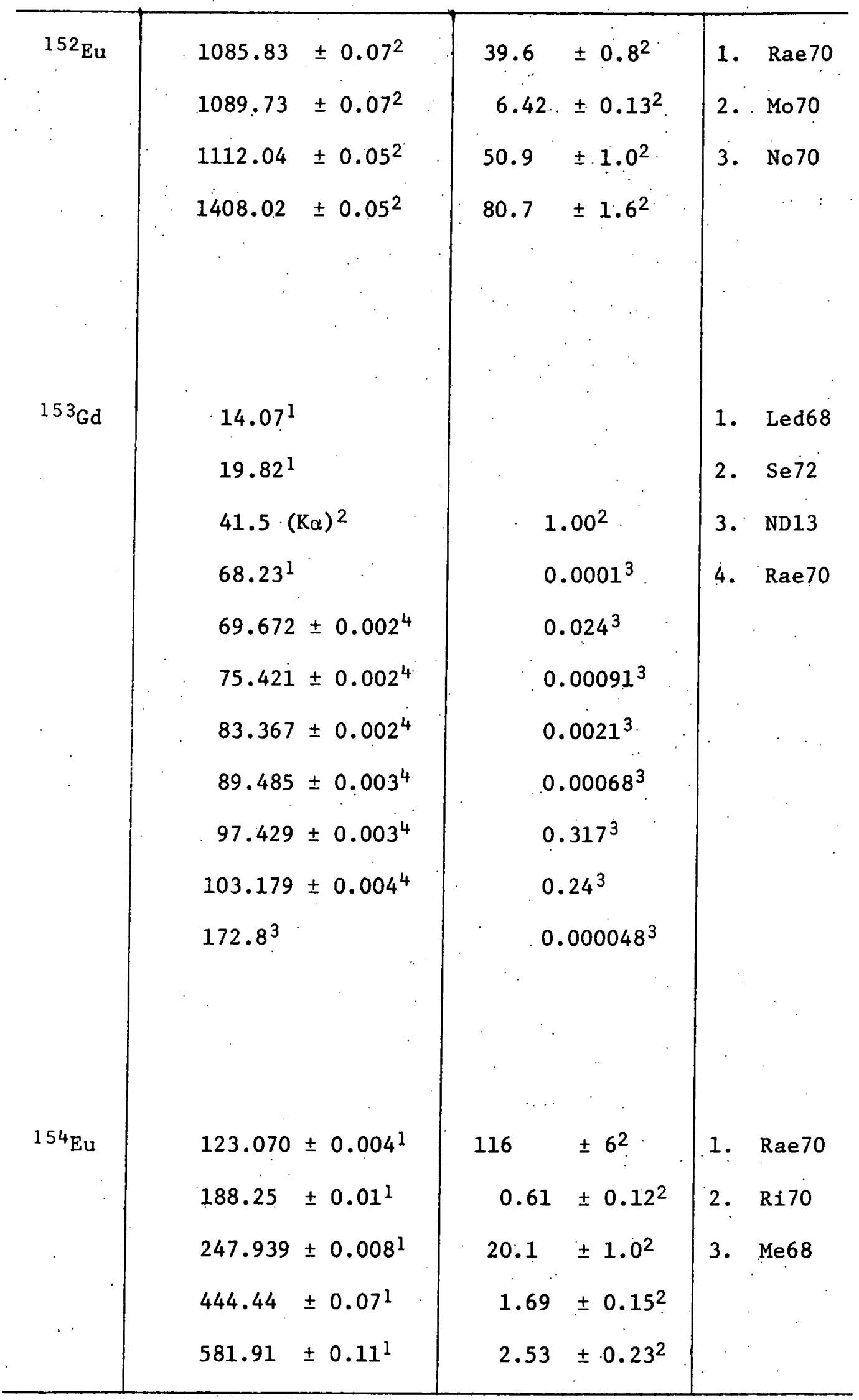




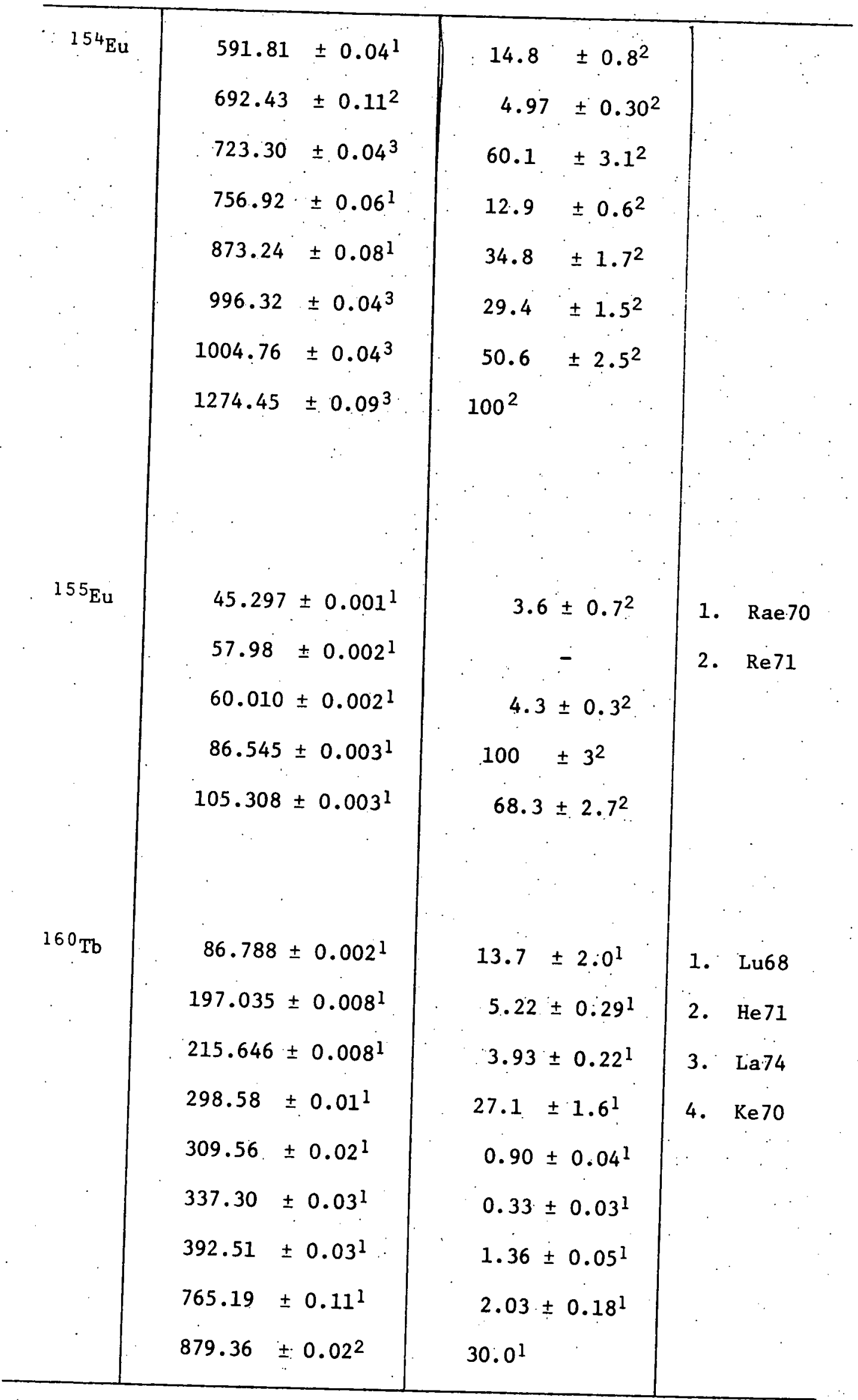




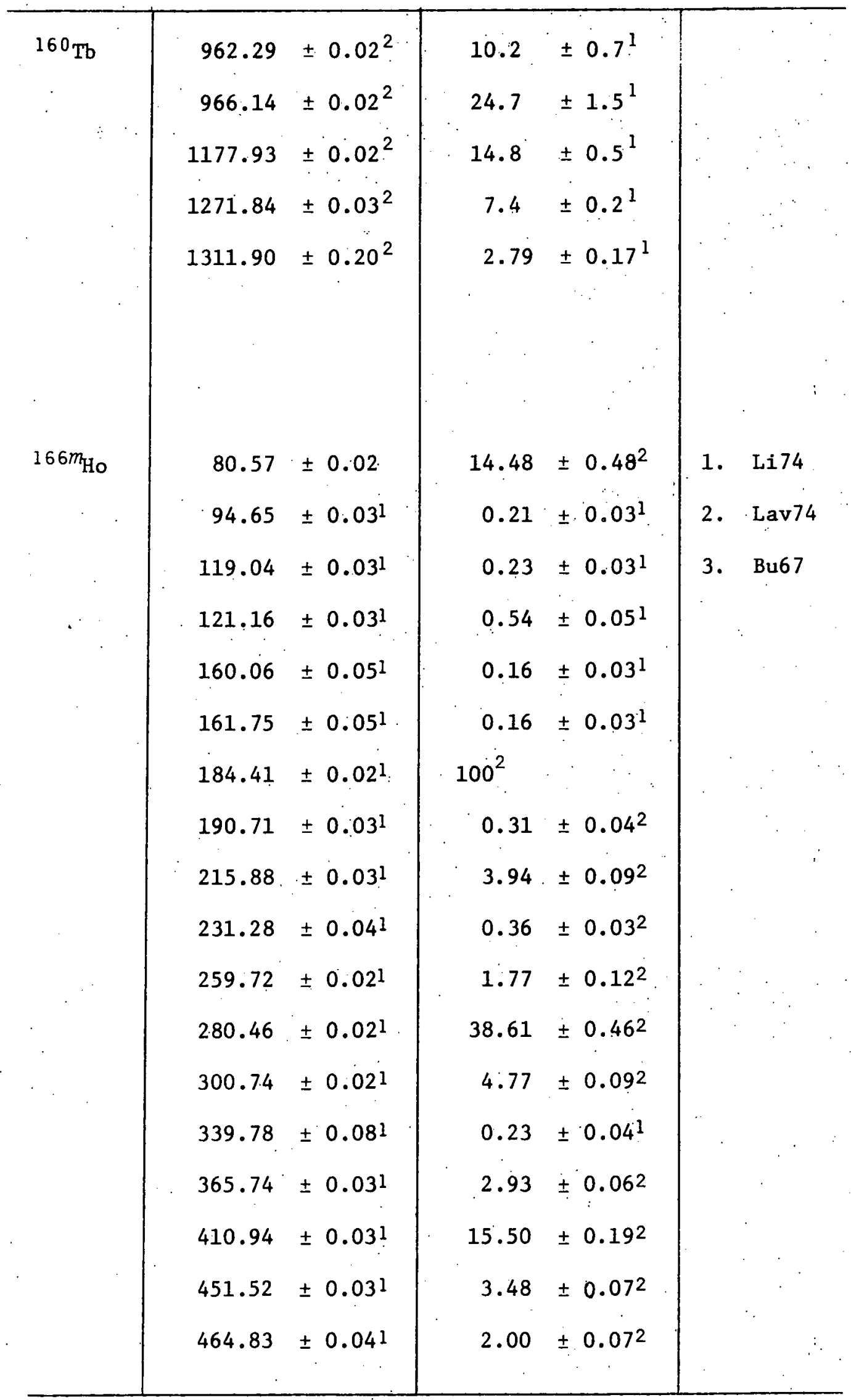




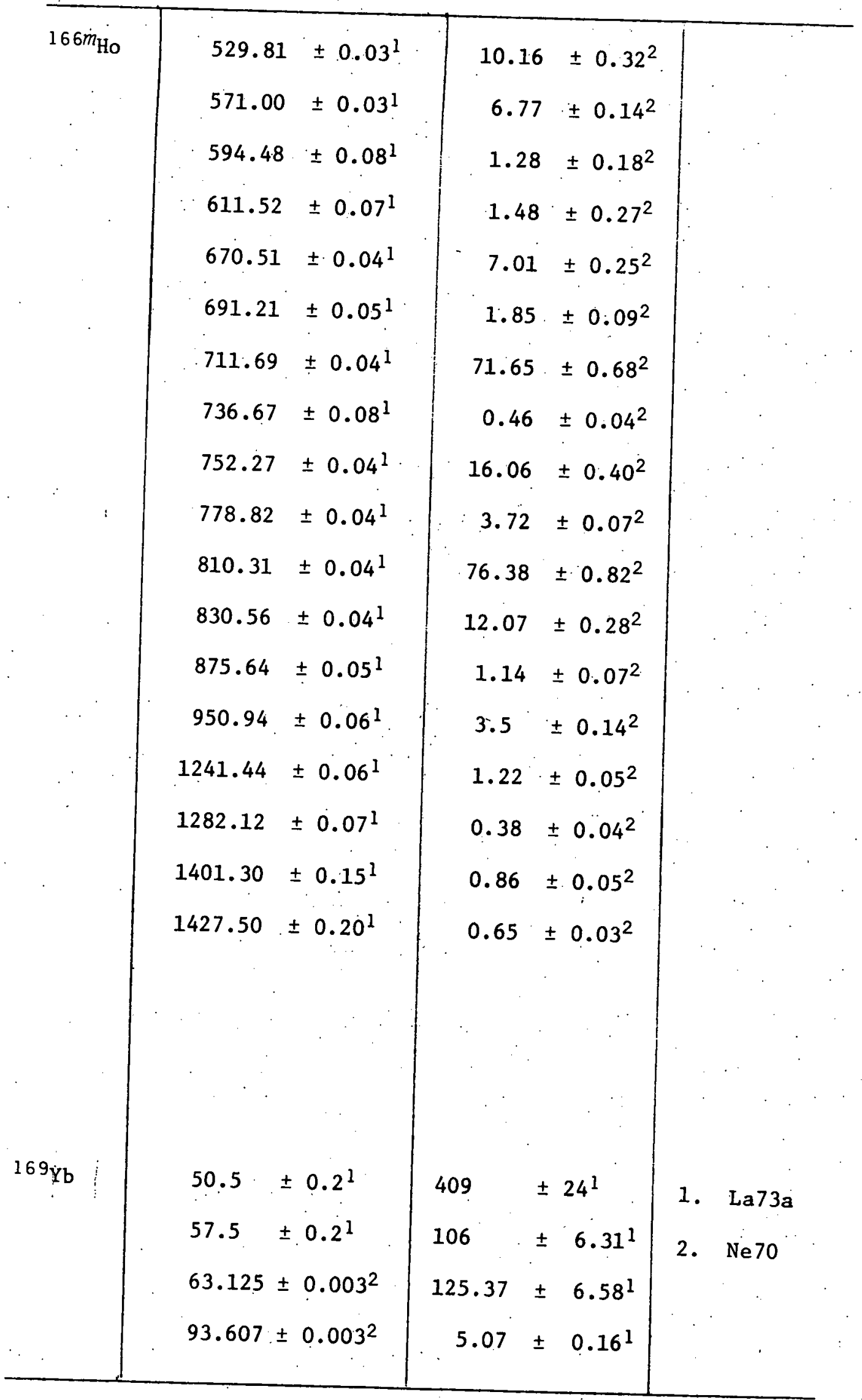




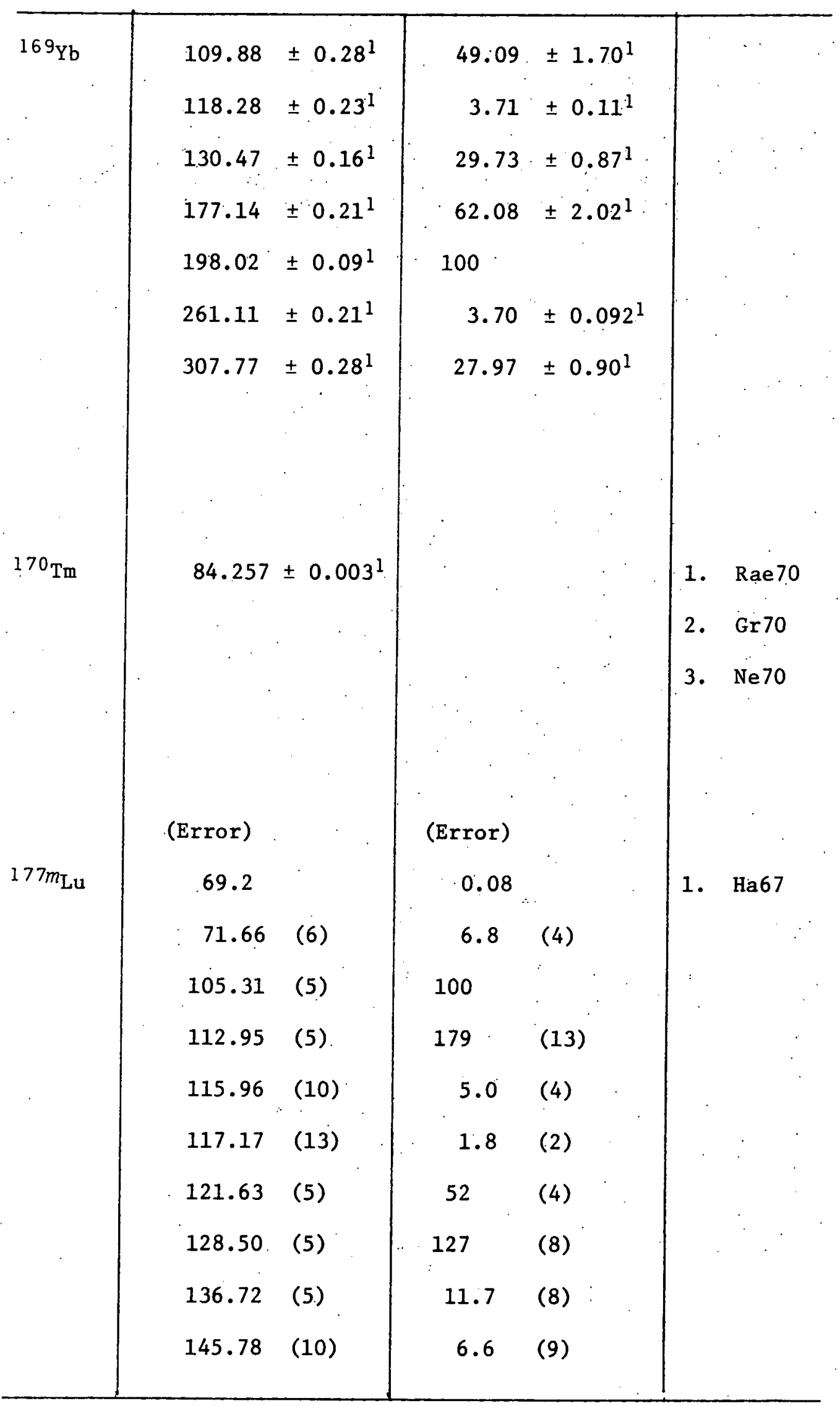




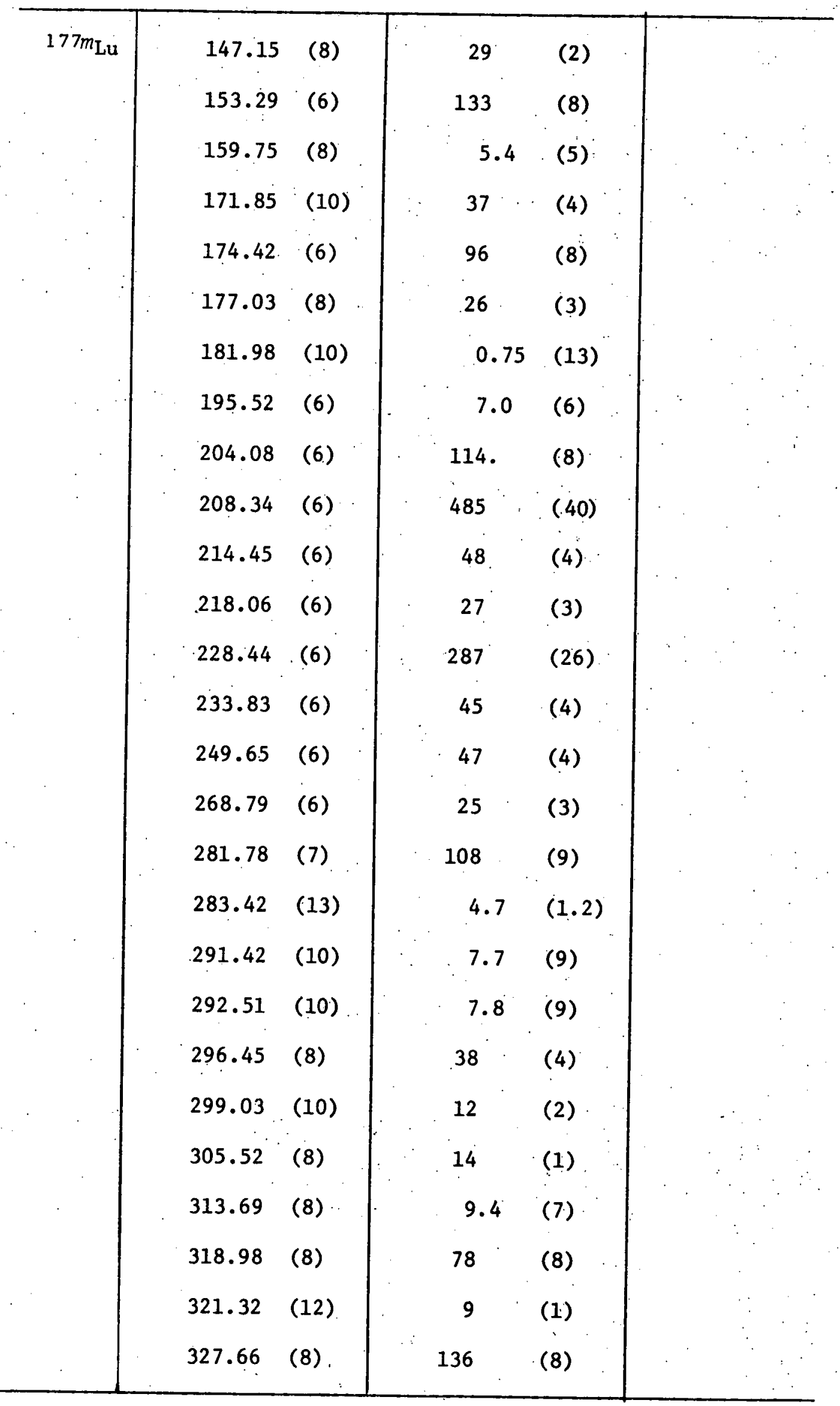




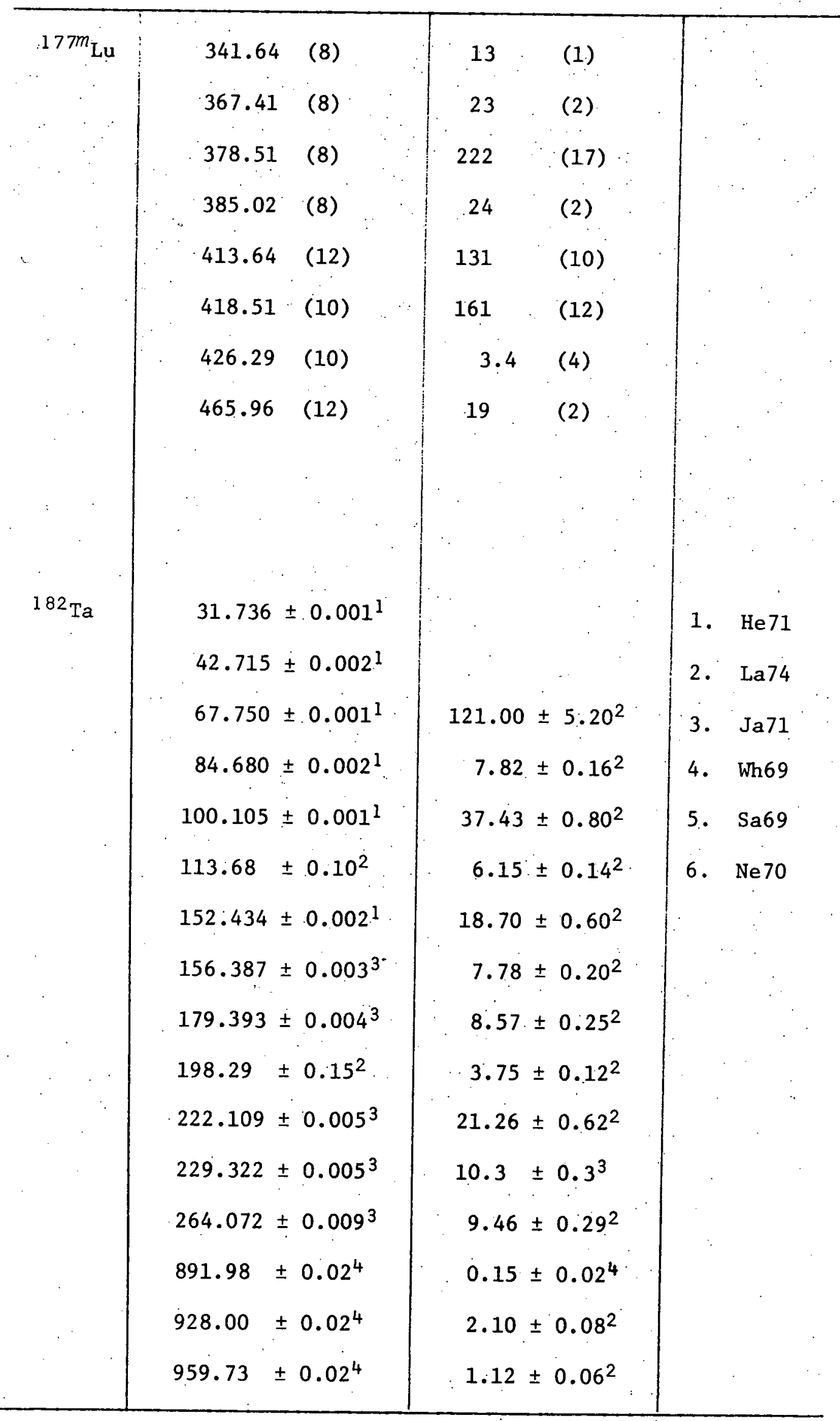




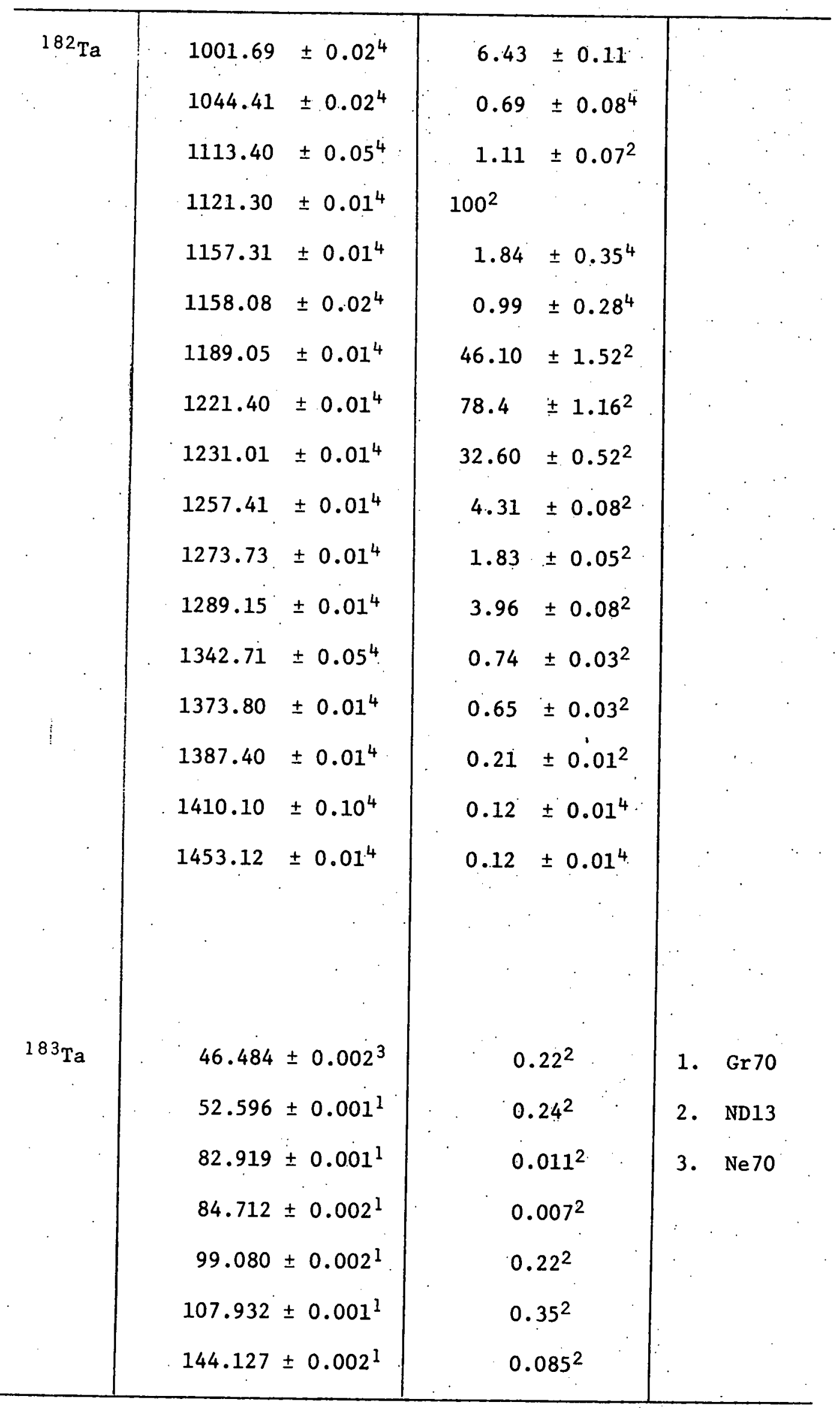




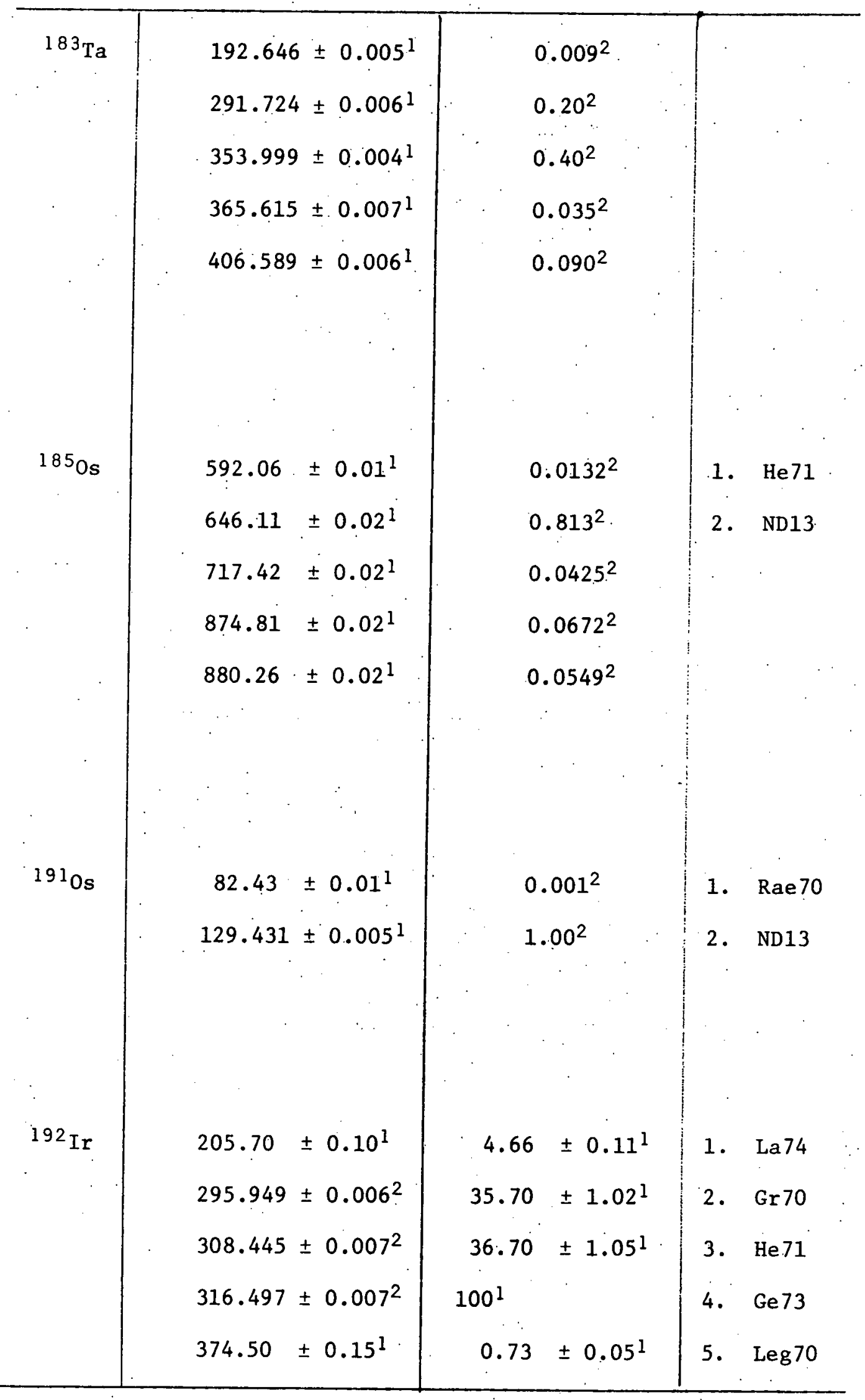




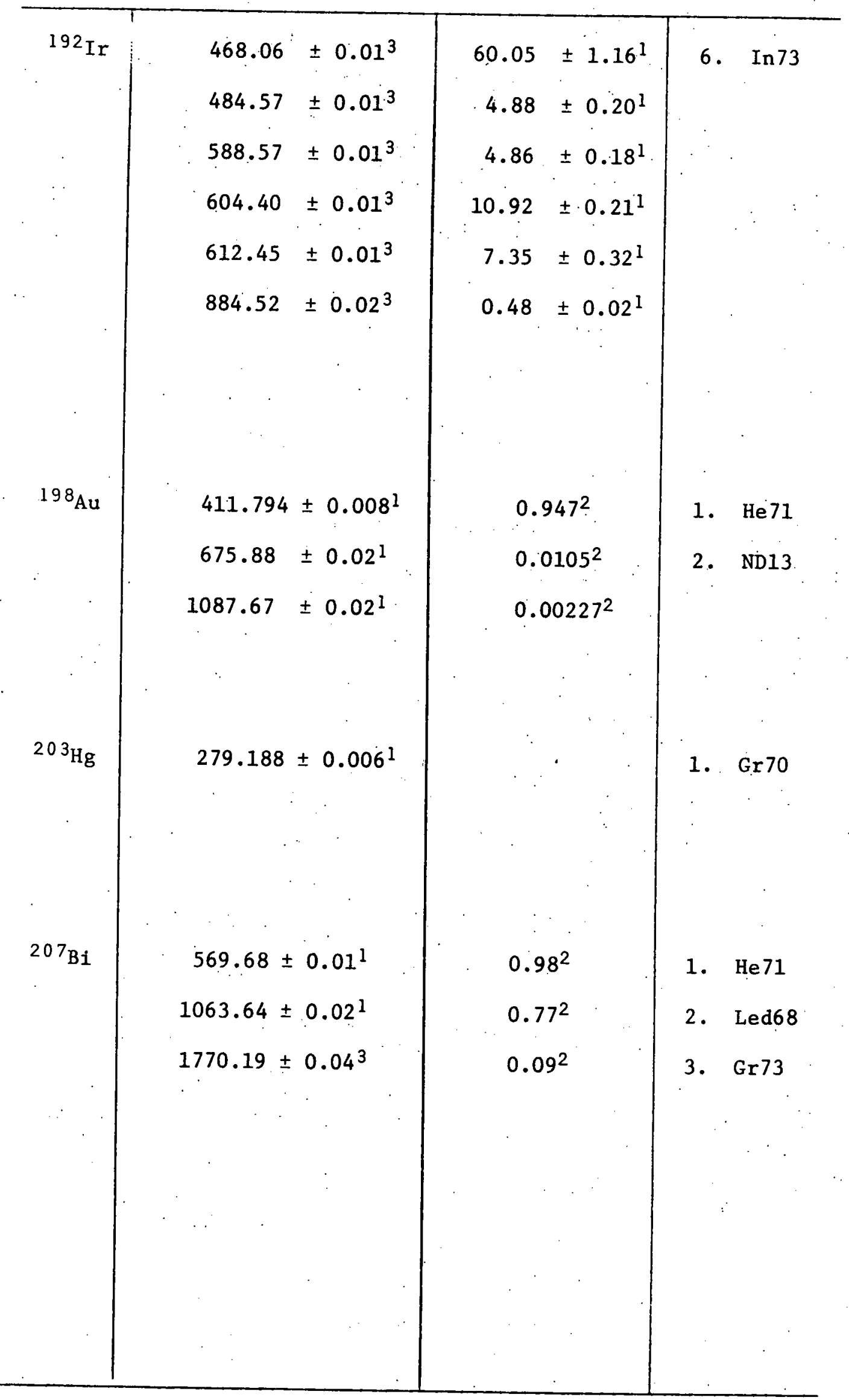




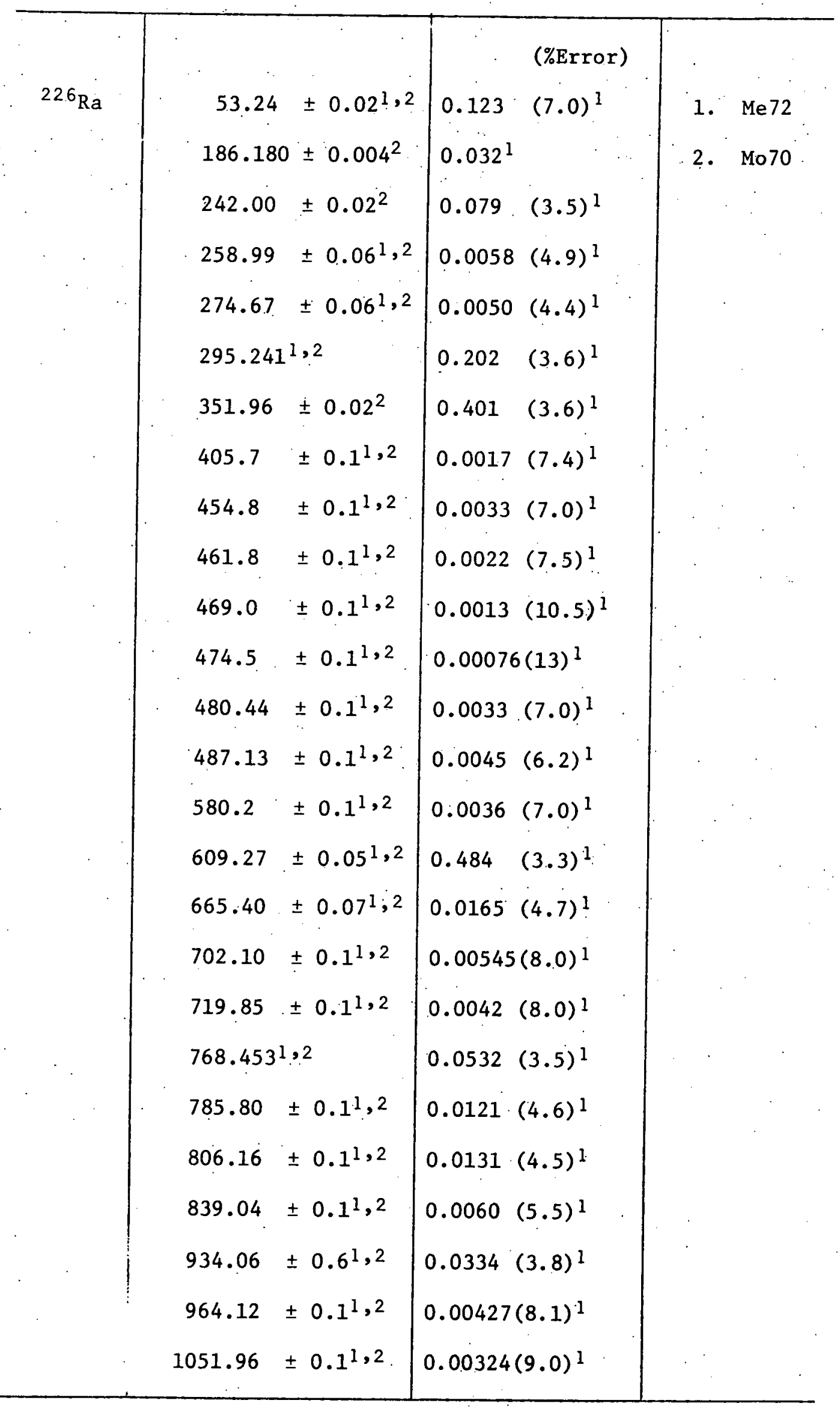




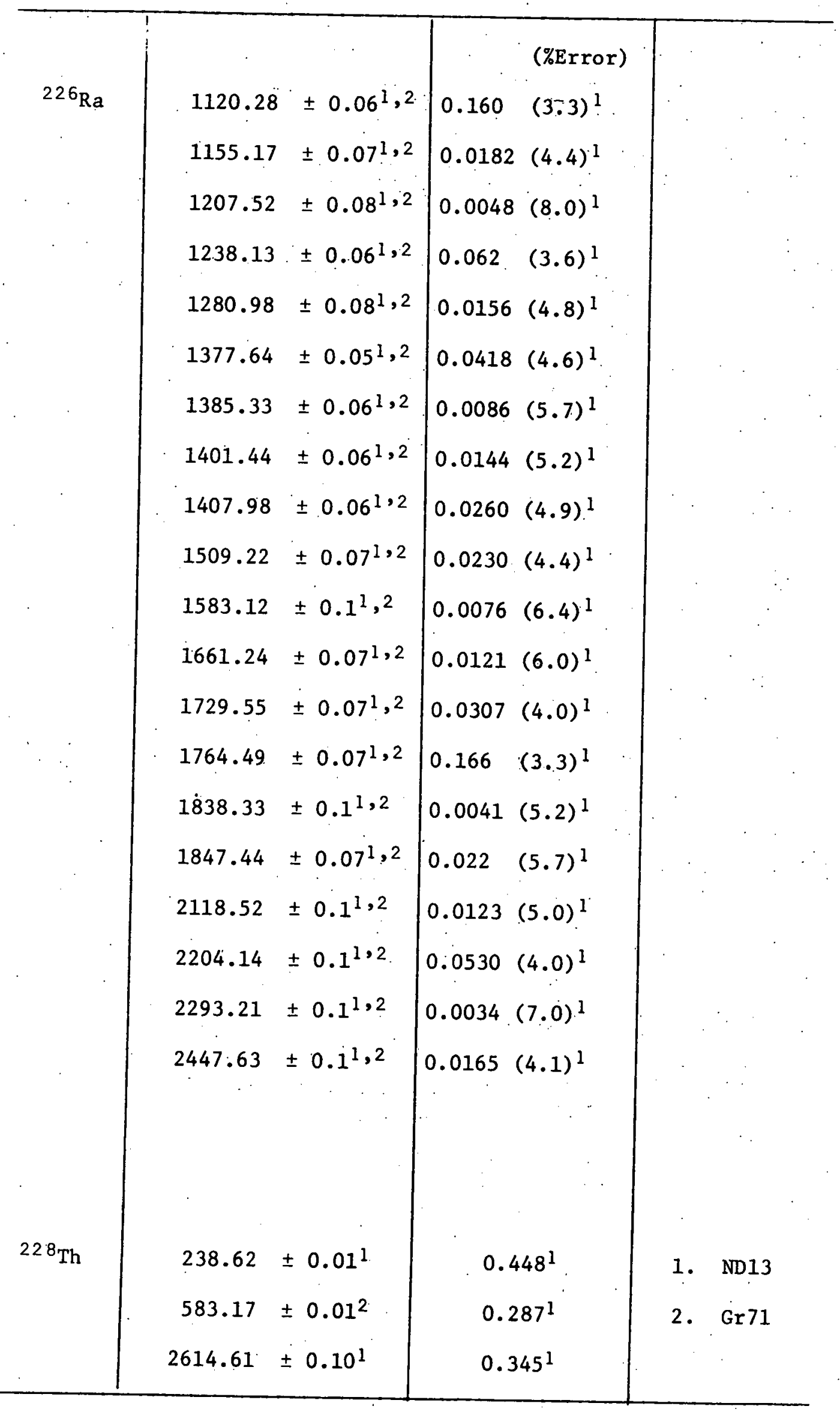




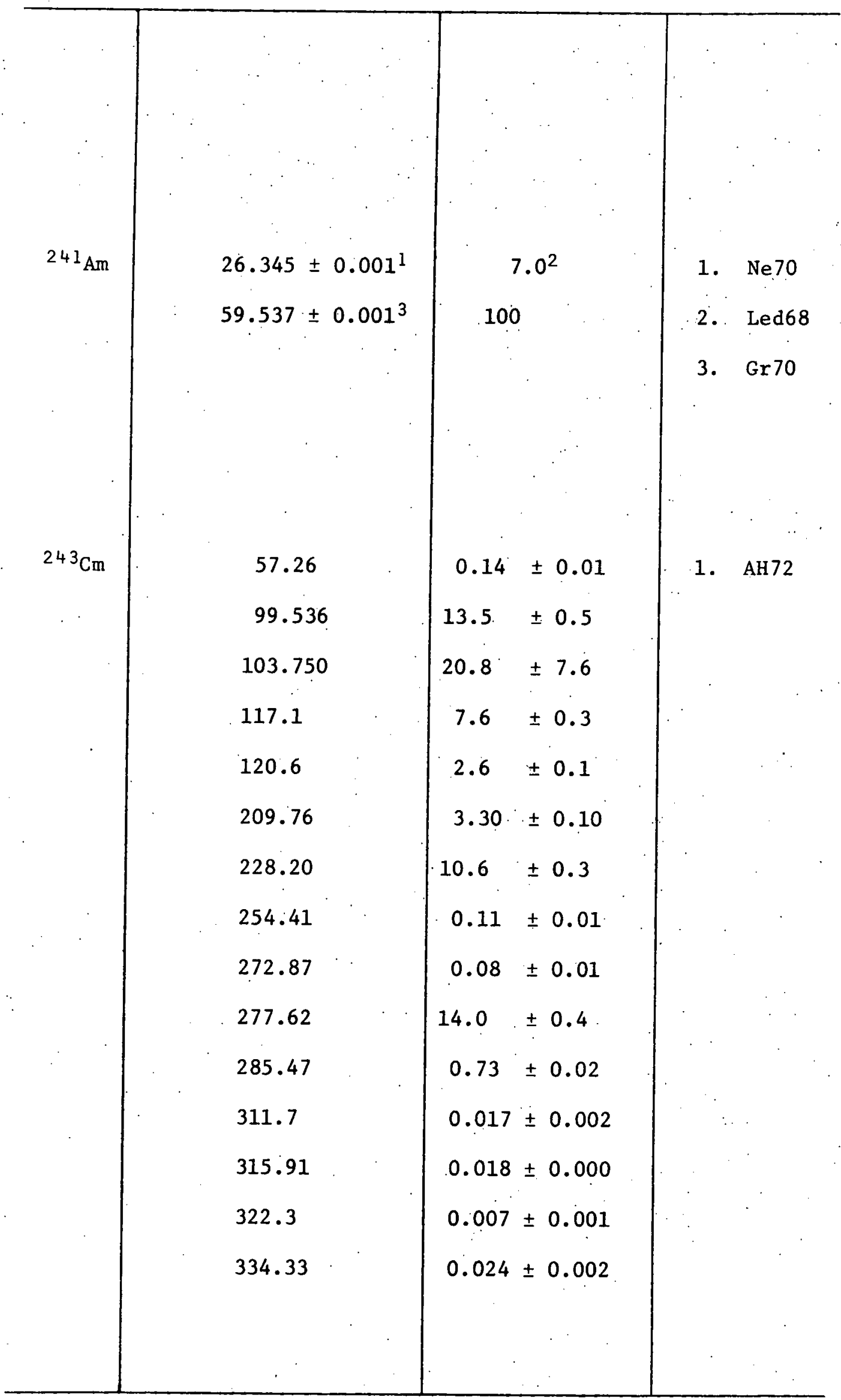


REFERENCES :

Ar74 G. Ardisson, S. Larib1, and C. Marsol, Nuc1. Phys. A223 (1974) 616 .

Ah71 : I. Ahmad and M. Wahlgren, Nucl. Instr. Meth. 99, (1972) 333.

Au69 F. P. Auer, J. J. Reidy, and M. L. Wiedenbeck, Nucl. Phys. A124 (1969) 199.

Bu67 S. B. Burson, P. F. A. Goudsmit, and J. Konijn, Phys. Rev. 158 (1967) 1161.

Ca71 D. C. Camp and G. L. Meredith, Nucl. Phys. A166 (1971) 349.

Ch67 C. Chasman, K. W. Jones, R. A. Ristinen, and D. E. Alburger, Phys. Rev. 159 (1967) 830.

Chi69 G. Chilosi, E. Eichler, and N. K: Aras, Nucl. Phys: Al23 (1969) 327.

Das73 B. K. Das Mahapatra and P. Mukherfee, Nuc1. Instr. Meth. 107 (1973) 611.

Ei68 C. W. E. Van Eijk, B. Van Nooljen, F. Schutte, S. M. Brahmavar, J. H. Hamilton, and J. J. P.inajlan, Nucl: Phys. Al21 (1968) 440.

Ga72 P. L. Gardulsk1 and M. L. Wiedenbeck, Nucl. Instr. Meth. 105

Ge71 R. J. Gehrke, J. E. Cline, and R. L. Heath, Nucl. Instr. Meth. 91 (1971) 349 .

Ge73 R. J. Gehrke, Nuc1. Phys. A204 (1973) 26. 
Gr70 R. C. Greenwood, R. G. Helmer, and R. J. Gehrke, Nuc1. Instr. Meth. 77 (1970) 141.

Gr73 R. G. Greenwood, R. G. Helmer, and R. J. Gehrke, unpublished.

Gu68 R. Gunnink, R. A. Meyer, J. B. Niday, and R. P. Anderson, Nuc1. Instr. Meth. 65 (1968) 26.

$\mathrm{Ha} 67$

$\operatorname{Ham} 71$

$\operatorname{Han} 73$

He 71

$\operatorname{In} 73$

$\mathrm{Ja71}$

$\operatorname{Ke} 70$

Ko71
A. J. Haverfield, F. M. Bernthal, and J. M. Hollander, Nucl. Phys. A94 (1967) 337.

J. H. Hamilton, S. M. Brahmavar, J. B. Gupta, R. W. Lide, and P. H. Stelson, Nucl. Phys. A172 (1971) 139.

A. Hanser, Nuc1. Instr. Meth. 107 (1973) 187.

R. G. Helmer, R. C. Greenwood, and R. J. Gehrke, Nucl. Instr. Meth. 96 (1971) 173.

H. Inoue and Y. Yoshizawa, Nucl. Instr. Meth. 108 (1973) 385.

L. J. Jardine, Nucl. Instr. Meth. 96, (1971) 259.

$\operatorname{La} 74$

N. Lavi, Nuc1. Instr. Meth. 116 (1974) 457.

La73a

N. Lavi, Nucl. Instr. Meth. 107 (1973) 197.

La 73b

N. Lavi, Nucl. Instr. Meth. 109 (1973) 265.

Led68

C. M. Lederer, J. M. Hollander, and I. Perlman, Table of Isotopes, 6 th ed., John Wiley \& Sons, Inc. (1968). 
Leg68 Legrand, J. P. Boulanger, and J. P. Brethon, Nucl. Phys.
Al07 (1968) 177.

Leg70 J. Legrand, J. Morel, and C. Clement, Nuc1. Phys. A142 (1970) Li74 W. A. Lingeman, F. W. N. De Boer, and B. J. Meijer, Nucl.
Instr. Meth. $118(1974) 609$.

Lu68 M. A. Ludington, J. J. Re1dy, M. L. Wiedenbeck, D. J. McMillan, J. H. Hamilton, and J. J. Pinajian, Nucl. Phys. A119 (1968)
398.

\author{
MeN73 L. A. McNelles and J. L. Campbe1l, Nucl. Instr. Meth. 109 (1973)
241 . \\ Me68 R. A. Meyer, Phys. Rev. 170 (1968) 1089. \\ Me 72 R. A. Meyer, unpublished. \\ Mo70 R. S. Mowatt, Can. J. Phys. 48 (1970) 2606.
}

ND13 Nuc1. Data Tables 13 (1972).

NDA8 Nuc1. Data Tables A8 (1970).

Ne70 9. C. Nelson and B. G. Saunders, Nucl. Instr. Meth. 84 (1970)

No70 A. Notea and E. Elias; Nucl. Instr. Meth. 86 (1970) 26.9.

Phe 70 M. E. Phelps, D. G. Sarantites, and W. G. Winn, Nucl. Phys.
Al49. (1970) 647.

Ghi72 B. Phillips, S. M. Brahmavar, J. H. Hamilton, and T. Kracikova,
Nuc1. Phys. Al82 (1972) 606.

Pr71 W. W. Pratt, Nuc1. Phys. Al70 (1971) 223. 